\title{
PLIF study of Mars Science Laboratory capsule reaction control system jets
}

\author{
C.T. Johansen ${ }^{*}$, P.M. Danehy ${ }^{\dagger}$, S.W. Ashcraft ${ }^{\ddagger}$, B.F. Bathel ${ }^{\S}$, J.A. Inman ${ }^{* *}$, S.B. Jones ${ }^{\dagger \dagger}$ \\ NASA Langley Research Center, Hampton VA, 23681-2199
}

\begin{abstract}
Nitric-oxide planar laser-induced fluorescence (NO PLIF) was used to visualize the flow in the wake of a Mars Science Lab (MSL) entry capsule with activated reaction control system (RCS) jets in NASA Langley Research Center's 31-Inch Mach 10 Air Tunnel facility. Images were processed using the Virtual Diagnostics Interface (ViDI) method, which brings out the three-dimensional nature of the flow visualization data while showing the relative location of the data with respect to the model. Comparison of wind-on and wind-off results illustrates the effect that the hypersonic crossflow has on the trajectory and structure of individual RCS jets. The visualization and comparison of both single and multiple activated RCS jets indicate low levels of jet-jet interaction. Quantitative streamwise velocity was also obtained via NO PLIF molecular tagging velocimetry (MTV).
\end{abstract}

\section{Introduction}

$\mathrm{T}$ HERE has been recent interest at NASA in the performance and reliability of the reaction control system (RCS) to provide sufficient control authority for the Mars Science Laboratory (MSL) entry vehicle. ${ }^{1,2,3}$ Figure 1 shows rendered side- and back-view schematics of the MSL aeroshell and thermal protection system, including the RCS jet configuration distributed on the vehicle's aftbody. Values for key parameters indicated in Fig. 1 are listed in Table 1. Derived from the Viking mission design, the MSL is a lifting, capsule-type entry vehicle with a heat shield cone half angle of 70 degrees. Compared to previous Mars missions, MSL is the first entry vehicle to use a guided entry strategy and will be the largest and most massive vehicle yet sent to the planet surface. The MSL will experience high aero-thermal and structural loads during entry due to the presence of turbulent heating and due to the relatively high vehicle ballistic coefficient. ${ }^{4}$ Prior to the entry, descent, and landing phase (EDL), two $75 \mathrm{~kg}$ cruise balance masses (CBMs) will separate to alter the vehicle's center of mass, inducing an angle of attack (AoA) on the capsule during entry. ${ }^{5}$ The coordinated jet impulses from the RCS system facilitate a series of bank reversal maneuvers through the manipulation of the vehicle's lift vector. Increasing the vehicle's AoA through separation of the cruise balance masses enhances the lift-to-drag (L/D) ratio, which both increases the effectiveness of the bank reversal maneuvers and allows the vehicle to reduce momentum at higher altitudes. When the vehicle is orientated at a 16 degree AoA at Mach 24, for example, the nominal lift-to-drag ratio is approximately L/D 0.24. The control authority enables the vehicle to fly a guided entry, controlling downrange and cross range through bank reversals. The controller also damps unwanted oscillations due to dynamic instabilities and atmospheric perturbations. A detailed overview of the EDL process, including deployment of the supersonic parachute and the powered descent process are discussed in the literature. ${ }^{5}$ Four RCS jet pairs are distributed and orientated on the aftbody to provide adequate pitch, roll, and yaw moments to the vehicle while minimizing interaction effects. ${ }^{1}$ Each nozzle provides approximately $290 \mathrm{~N}$ of thrust and operates intermittently in short bursts, lasting several seconds cumulatively in duration. $^{2}$

* National Institute of Aerospace Visitor in Advanced Sensing and Optical Measurement Branch, MS 493; also Natural Sciences and Engineering Research Council of Canada Post-doctoral Fellow.

${ }^{\dagger}$ Research Scientist, Advanced Sensing and Optical Measurement Branch, MS 493. AIAA Associate Fellow.

* USRP Student, Advance Sensing and Optical Measurement Branch, MS 493, also Student at University of Kentucky.

$\S$ Graduate Coop Student in Advanced Sensing and Optical Measurement Branch, MS 493; also Graduate Student, University of Virginia. AIAA Student Member

${ }^{* *}$ Research Scientist, Advanced Sensing and Optical Measurement Branch, MS 493. AIAA Member

${ }^{\dagger}$ Technician, Advanced Sensing and Optical Measurement Branch, MS 493 


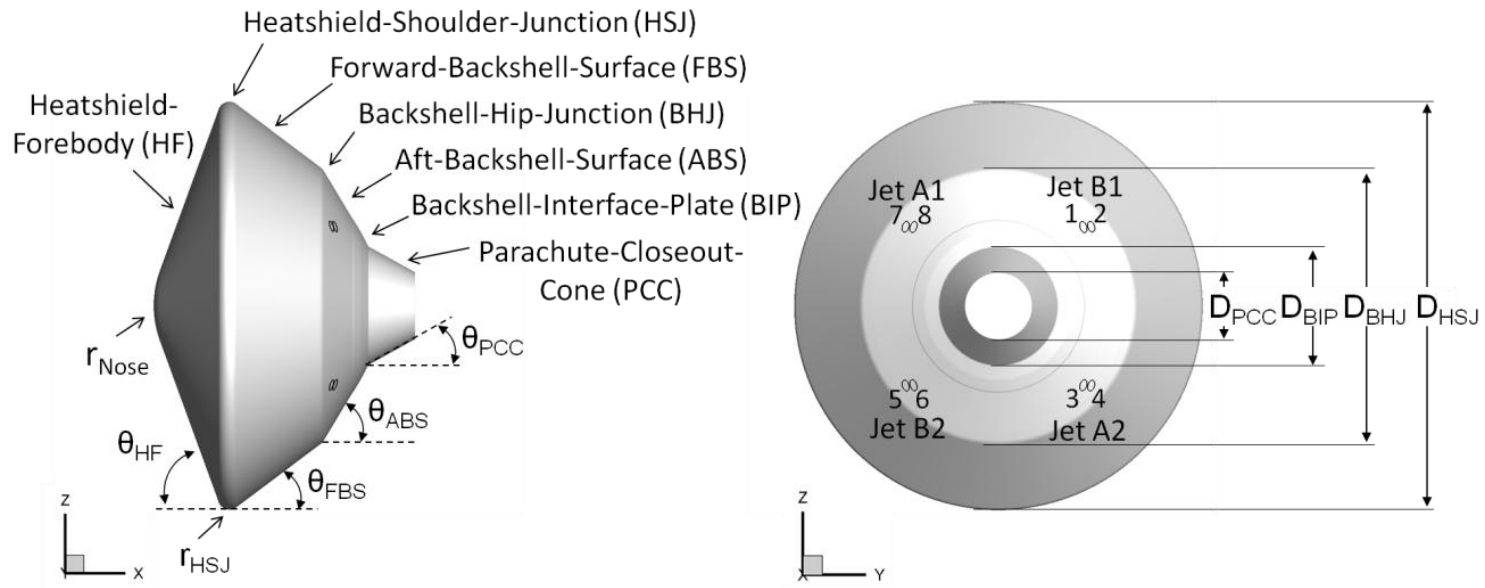

Figure 1: MSL aeroshell configuration showing side-view and back-view perspectives. Nomenclature and dimensions based on Ref. 2.

Table 1: Key parameters for MSL flight vehicle. Experimental model is 3.4\% scale of the flight vehicle.

\begin{tabular}{|c|l|l|l|l|l|l|l|l|l|l|}
\hline Parameter & $\Theta_{\mathrm{HF}}$ & $\Theta_{\mathrm{FBS}}$ & $\Theta_{\mathrm{ABS}}$ & $\Theta_{\mathrm{PCC}}$ & $\mathrm{r}_{\text {Nose }}$ & $\mathrm{r}_{\mathrm{HSJ}}$ & $\mathrm{D}_{\mathrm{HSJ}}$ & $\mathrm{D}_{\mathrm{BHJ}}$ & $\mathrm{D}_{\mathrm{BIP}}$ & $\mathrm{D}_{\mathrm{PCC}}$ \\
\hline Value & 70 & 37 & 59 & 29 & 1.125 & 12.5 & 4.5 & 3 & 1.3 & 0.74 \\
\hline Units & 0 & 0 & 0 & 0 & $\mathrm{~m}$ & $\mathrm{~cm}$ & $\mathrm{~m}$ & $\mathrm{~m}$ & $\mathrm{~m}$ & $\mathrm{~m}$ \\
\hline
\end{tabular}

During atmospheric entry the interaction between the hypersonic crossflow and the multiple RCS jets is not well understood. In the absence of RCS jets, several characteristic flow features are observed around the MSL vehicle when oriented at a high angle of attack (Fig. 2). Characteristic of blunt-body hypersonic flow, a bow shock forms ahead of the MSL vehicle, which is required to decelerate the approaching high Mach number, freestream gas. For a zero degree vehicle AoA, the stagnation point is located on the heatshield/forebody nose due to the symmetry of the flow. As the vehicle AoA increases, however, the stagnation point location migrates in the windward direction toward the capsule heatshield shoulder. The high stagnation temperature of the freestream gas ensures a distribution of high static temperature gas along the capsule heatshield forebody, driving the rate of heat transfer due to convection. From the stagnation point, the gas expands outward in the radial direction towards the edge of the capsule's heatshield, causing both the local Mach number and Reynolds number to increase. The gas transitions from subsonic to supersonic flow at the sonic line, the location of which is influenced by the vehicle AoA. On the windward side of the vehicle forebody, the sonic line forms near the heatshield shoulder. The asymmetry of the flow allows gas to accelerate to higher speeds along the leeward side of the heatshield forebody, which results in the positioning of the sonic line closer to the capsule nose. The small shoulder angle ensures that the sonic line and stagnation point remain on the vehicle forebody, even for high vehicle AoA (e.g. 26 degrees). ${ }^{4}$ The positioning of the sonic line influences the location of peak-heating on hypersonic blunt-body vehicles.

Numerical simulations have been performed to study the effects of laminar-to-turbulence transition on the vehicle's heating loads. ${ }^{6,7}$ At peak heating conditions with the vehicle AoA at 16 degrees, the majority of flow along the windward side of the heatshield forebody remains subsonic and laminar. Based on the local momentum thickness Reynolds number, transition to turbulence is expected to occur near the windward shoulder junction and on the leeward heatshield forebody. Compared to a theoretical laminar heating load solution, transition to turbulence is expected to increase the peak heating loads on the heatshield forebody by a magnitude of $2.5 .^{6}$

At both windward and leeward shoulder junctions a supersonic expansion fan turns the gas from the forebody to the aftbody. Depending on the turning angle, the sudden expansion can cause flow separation on the aftbody and a shear layer, or mixing layer, to form between the outer flow and a recirculation zone. Downstream of the capsule shoulder junction, a lip shock (not shown in the figure) redirects the outer flow away from the aftbody. Finally the outer flow is processed by a recompression shock wave, turning the gas downstream. The size of the recirculation zone is sensitive to the vehicle AoA, but is also influenced by other factors such as local levels of turbulence. ${ }^{8}$ 
Transition to turbulence along the vehicle forebody or within the developing shear layer enhances the transport of high momentum outer flow towards the vehicle aftbody surface, which can suppress or delay flow separation. ${ }^{8}$

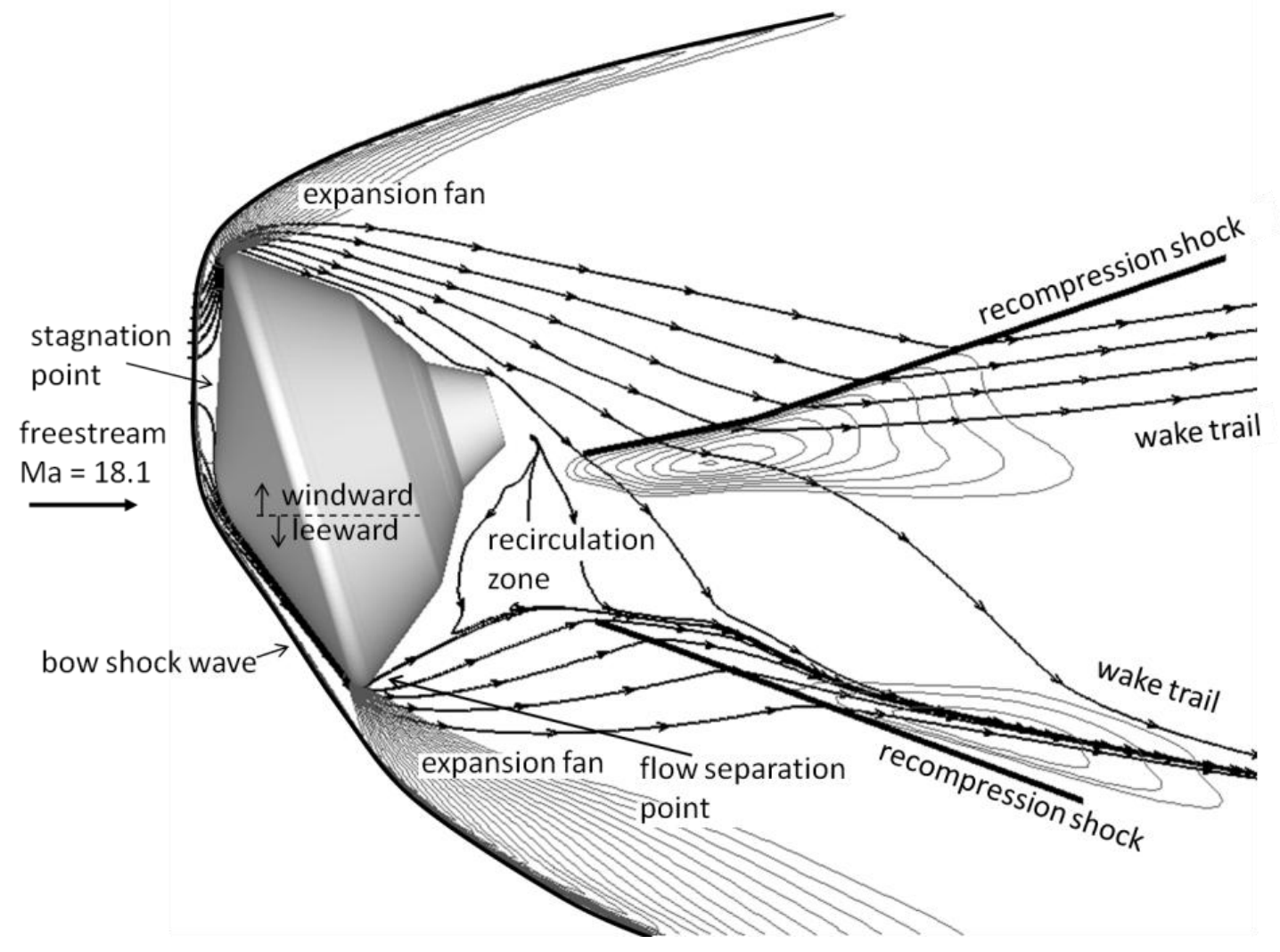

Figure 2. Hypersonic flow field around MSL vehicle. Numerical predictions of stream traces (thick lines with arrows) and selected iso-lines of pressure are overlaid onto schematic of vehicle (adapted from Ref. 1).

There are many potential mechanisms that could promote transition to turbulence including surface roughness, ablation, or jet-in-crossflow. Flow separation along the aftbody of a hypersonic blunt-body re-entry type vehicle is known to affect aerodynamic moments due to an asymmetric pressure field distribution on the vehicle surface. ${ }^{9}$ Furthermore, the unsteady aerodynamic effects that turbulence has on these separated regions are known to increase the dynamic instability for these types of vehicles. ${ }^{10}$ For large vehicle AoAs, flow on the windward side of the model can stay attached to the model surface due to the small effective turning angle (Fig. 2). On the leeward side, however, the large expansion around the capsule shoulder junction leads to a large separated region on the vehicle aftbody that is comprised mostly of subsonic flow. After the gas is processed by a recompression shock wave behind the vehicle, a wake trail is formed.

In addition to the aforementioned flow structures around a blunt-body shape, the presence of under-expanded RCS jets on the vehicle's aftbody adds another layer of complexity to the flow. Review of an isolated jet-incrossflow is useful for the interpretation of the results presented later. Figure 3 shows side and isometric views of an under-expanded jet in a supersonic crossflow, obtained from the literature. ${ }^{11,12}$ A bow shock wave forms ahead of the jet due to the low streamwise momentum of the jet fluid relative to the crossflow. The interaction of the bow shock wave with the subsonic portion of the upstream boundary layer causes the flow to separate. Downstream of the bow shock wave, spanwise components of vorticity in the boundary layer roll up around the jet, forming a horseshoe vortex. At the lip of the jet orifice, a Prandtl-Meyer expansion of the jet gas occurs due to the large jet-tocrossflow pressure ratio. The jet gas is subsequently compressed by a barrel shock wave and later by a Mach disk. A shear layer forms between the barrel shock wave and the bow shock wave, providing the mechanism for the production of turbulence. A counter-rotating vortex pair is shown in the isometric view of Fig. 3. The vortex pair interacts with the boundary layer and convects high momentum, high temperature gas from the freestream down to the wall surface. 
(a)

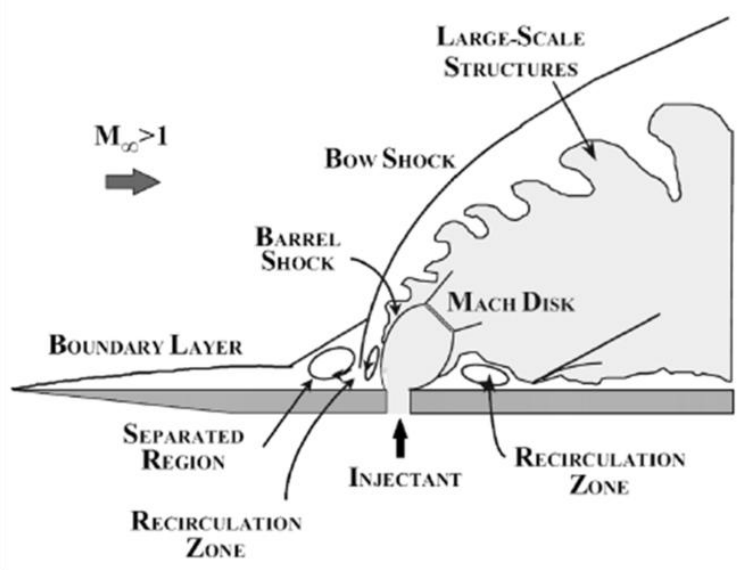

(b)

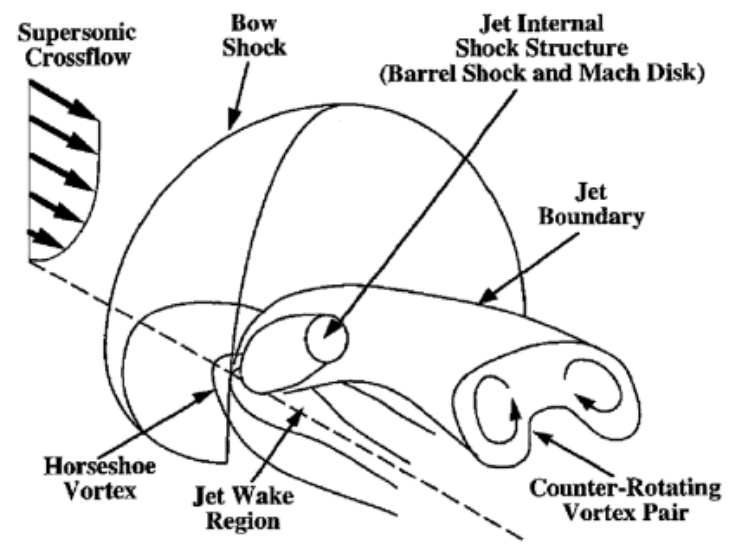

Figure 3. Schematics of an under-expanded supersonic jet in crossflow. The side-view (a) is an instantaneous snapshot of the flow field (Ref. 11). The isometric view (b) is a time-averaged sketch of the flow field (Ref. 12).

There are a few key differences between the jet interaction with the MSL flow field and a jet oriented normal to the cross-flow as shown in Fig. 3. First, the RCS jets are oriented at angles to crossflow (scarfed) rather than being orientated normal to the surface and the crossflow. This orientation allows the RCS to achieve yaw, pitch, and roll moments depending on the combination of jets used. Second, some of the RCS jets are injected into regions of separated flow instead of an idealized attached boundary layer flow. For low AoA, both the windward (A1, B1) and leeward (A2, B2) side RCS jets interact with a separated, subsonic crossflow. For higher AoA, the windward side jets interact with an attached, supersonic crossflow. Third, each RCS jet location includes two closely spaced nozzles, which forces aerodynamic interference between jets to occur just downstream of the nozzle exit. Although both nozzles are oriented in the same direction, the barrel shock waves that form interact within a nozzle diameter.

Such aforementioned conditions make it possible for the resultant jets to impinge on the capsule surface or collide with other jets. A local increase in surface pressure can alter the capsule forces and moments, while an increase in surface temperature can damage the vehicle's thermal protection. In a numerical study of the MSL RCS jets, it was found that jet impingement on the capsule surface causes an increase in heat flux compared to the jet-off condition. ${ }^{1}$ The jets can also influence the entire capsule wake flowfield and cause global changes to the pressure field. This unwanted effect is partially mitigated by orientating each jet vector closely with the crossflow vector and positioning the jets downstream on the vehicle aftbody near the parachute closeout cone (PCC). ${ }^{1}$

The extent of the interaction between the four different jet pairs is a current topic of investigation. ${ }^{1-3,6,13}$ Although the jet configuration was chosen to minimize adverse effects of these interactions on the capsule aerodynamics and aerothermodynamics, an experimental data set is needed to help validate the numerical models. The study of this type of problem is also valuable to manned spaceflight as capsule-type geometries have historically been used as a means of atmospheric reentry. This study builds on the expertise gained in using nitric-oxide planar laser-induced fluorescence (NO PLIF) to study a similar capsule-type flow in support of the Orion crew module (OCM). ${ }^{14,15}$ Similar to MSL, OCM also uses RCS jets to steer the vehicle during entry. In this study, a series of NO PLIF images are obtained through the translation of a laser sheet across the flow field and analyzed with the Virtual Diagnostics Interface (ViDI) method to visualize the RCS jet and MSL wake flow structures.

\section{Wind Tunnel, Model, and Experimental Setup}

Experiments studying the near wake flow, jet-crossflow interactions, and jet-jet interactions of the MSL vehicle were performed in NASA Langley Research Center's 31-Inch Mach 10 Air Tunnel facility. By electrically heating compressed air, the facility is able to provide Mach 10 flow through the 31-inch square test section for approximately 90 seconds. ${ }^{16}$ The test section has optical access through two large windows located on the top and bottom tunnel surfaces. A third smaller window is located on the side wall opposite to the model injection system. Each window transmits ultraviolet light, which allows the projection of the laser sheet through the top window into the test section. Images of the NO fluorescence were obtained through the side window. The nominal stagnation pressure and temperature produced by the facility was 1300 psi and 1325 degrees Fahrenheit, respectively. In order 
to study the effects of jet-crossflow interaction, some of the tests were performed in a quiescent flow (no crossflow) with the static pressure maintained at $0.1 \mathrm{psi}$, which is approximately two times larger than the aft-body static pressure measured during tunnel runs.

Figure 4 shows the side view of the approximately $3.4 \%$ scale titanium MSL entry capsule model. As shown, the capsule is at an AoA of -20 degrees with respect to the flow. This angle corresponds to a horizontal sting. A watercooled force and moment balance was located inside the capsule and aligned with the model's center of gravity. The balance cabling, thermocouple cabling, and $\mathrm{N}_{2} / \mathrm{NO}$ plumbing were protected from aerodynamic heating during the $\sim 90$ second runtimes by a protective shroud. The sting AoA was adjusted during the run using a motorized control system.

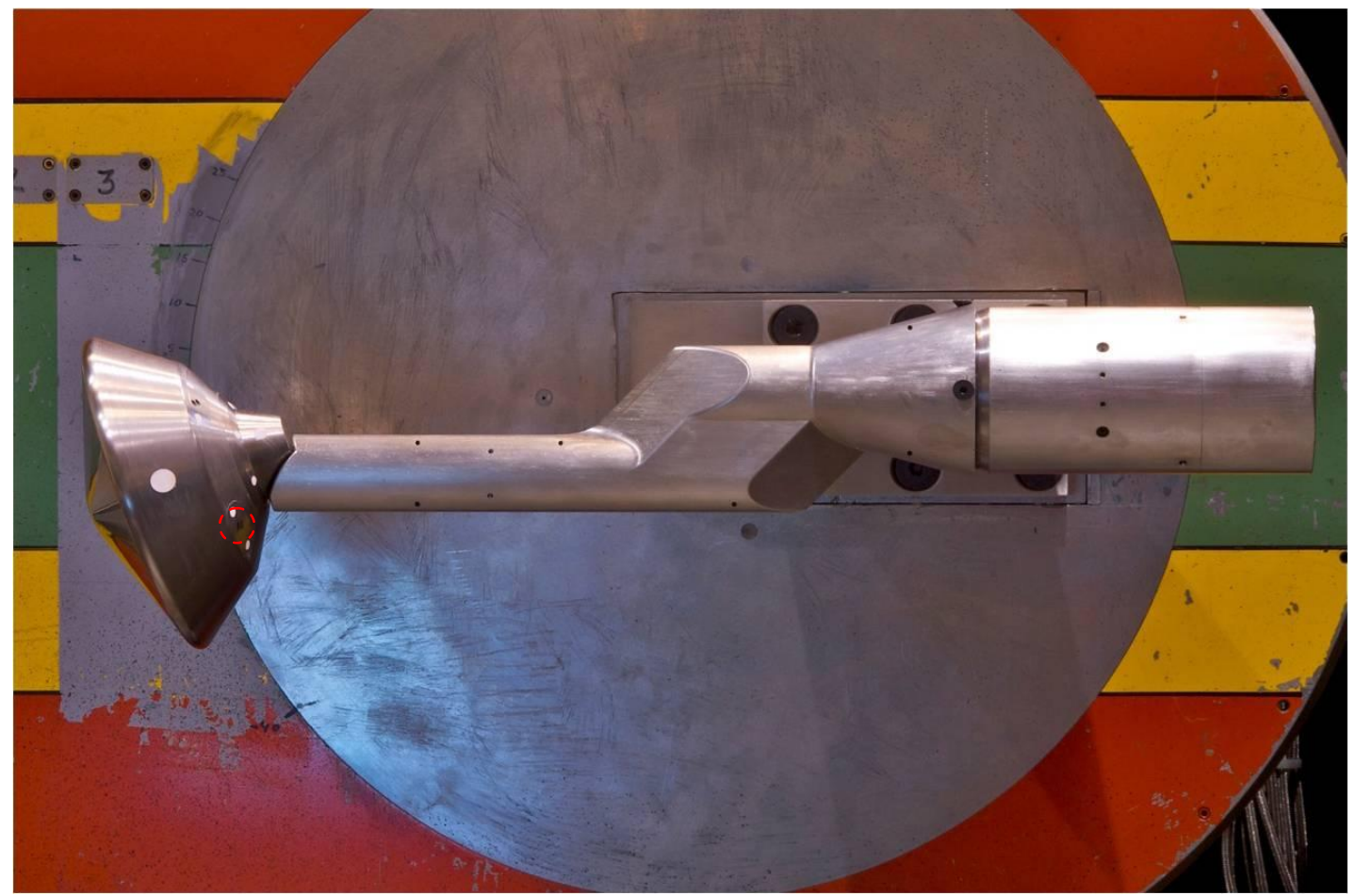

Figure 4: Side view photograph of MSL model, sting with protective heat shroud, and tunnel side wall. Dashed circle indicates location of RCS Jet B2, a schematic of which is shown in Fig. 5.

During some of the tests involving NO PLIF, the A1 and B2 RCS jets were operated. As on the flight vehicle, the RCS jets are distributed along the model aft-body in pairs. In all cases, both jets of the pair were operated at the same time. A schematic of one of the RCS jet pairs (B2) from the experimental model is shown in Fig. 5. Unlike the MSL vehicle body, the internal structure of the model's RCS jets is not directly scalable to the flight vehicle's jet geometry. The jet expansion ratio was altered to match similarity parameters and the model's nozzles were machined in a conical shape to reduce manufacturing complexity. In addition, the plenum cylinder was also machined flush with the throat cylinder. In contrast, the RCS system on the flight vehicle consists of smoothly varying converging-diverging nozzles. The scarfed interface between the conical RCS jet nozzles and the MSL backshell result in an elliptical nozzle exit shape. For clarity, a coordinate system based on the RCS jets is used so that proper side-, back-, and top-views of the jet pair could be shown in the figure. Both coordinate systems relative to the MSL capsule used in Fig. $1(x, y$, and $z)$ and relative to the RCS jet pair $\left(x^{\prime}, y^{\prime}\right.$, and $\left.z^{\prime}\right)$ are shown in the schematic. The nozzle spacing and the nozzle half angle are $\mathrm{W}_{\mathrm{RCS}}=0.128$-in and $\Theta_{\mathrm{RCS}}=12^{\circ}$, respectively. 

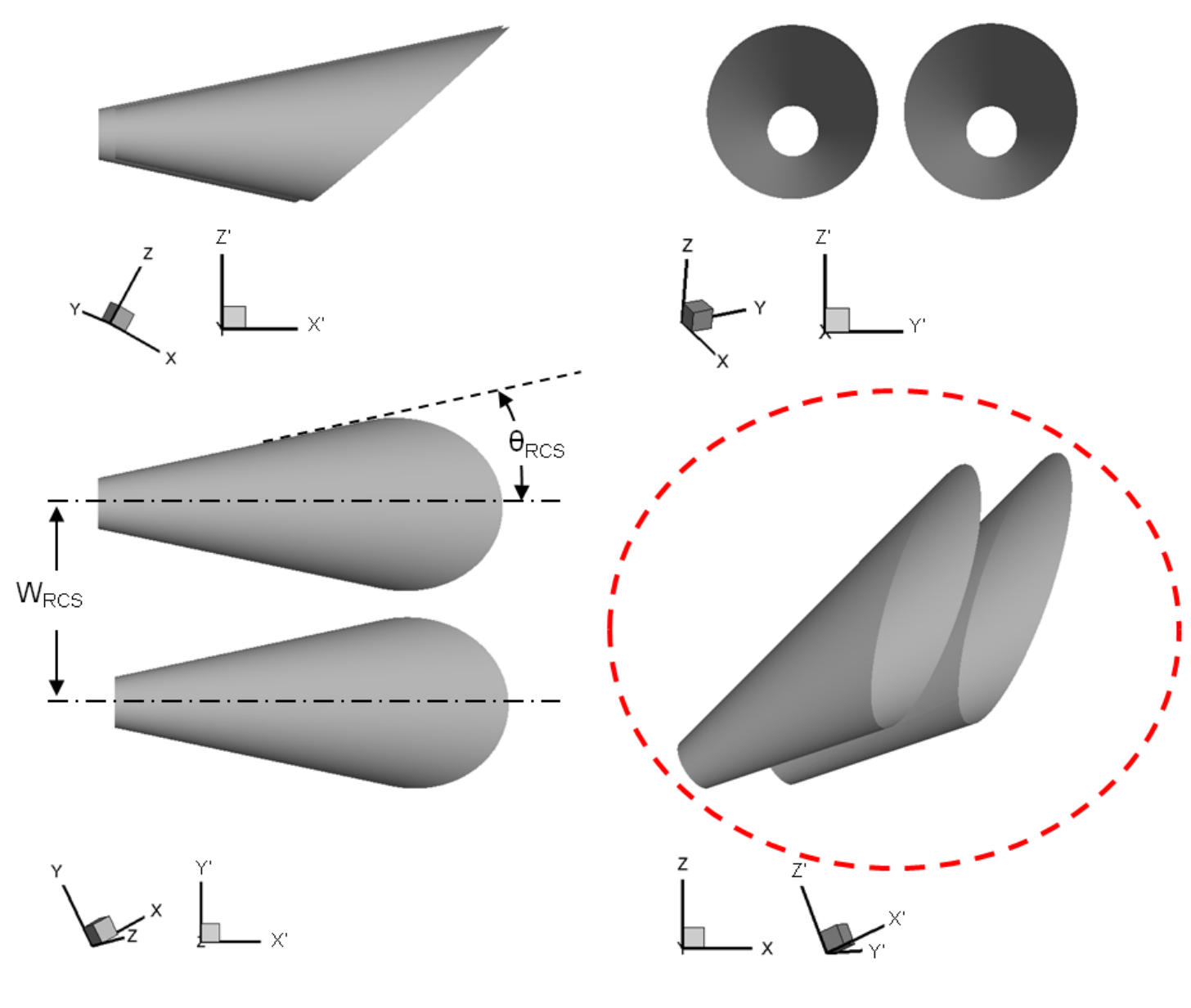

Figure 5: Experimental model RCS Jet B2 configuration showing side-view, top-view, and back-view perspectives. Coordinate system relative to RCS Jet is shown as primed coordinates (i.e. $x^{\prime}, y^{\prime}$, and $z^{\prime}$ ). Primed coordinates rotated by $\Theta_{x}=-4^{\circ}, \Theta_{y}=28^{\circ}$, and $\theta_{z}=30^{\circ}$. Red dashed circle corresponds to region of interest shown in Fig. 4.

For most of the runs, NO was seeded into the flow only through the RCS jet. The RCS jet fluid was seeded with $2-5 \% \mathrm{NO}$, with the balance being $\mathrm{N}_{2}$. This allowed observation of the shape, structure and trajectory of the RCS jets. A bottle of pure NO, initially at a pressure of $500 \mathrm{psi}$, was used to supply the NO for all runs. The NO delivery system is only rated for pressures less than $100 \mathrm{psi}$, so it could not directly supply the pressures (or flow rates) required for the experiment. To obtain the higher jet chamber pressures and flow rates required for this test, a 3.8 liter mixing chamber/plenum, which was external to the tunnel, was used. A regulator on the NO bottle, was adjusted to about 30 psi. This 3.8 liter plenum was first filled with $6.1 \pm 0.3$ psi of pure NO by flowing the pure NO at $1000 \mathrm{sccm}$ for 95 seconds. This method was chosen instead of using a pressure gauge because it is more accurate than using any of the other pressure gauges available in the system (the lowest of which is a mechanical gauge that reads up to $100 \mathrm{psi}$ and is probably accurate to $\pm 5 \mathrm{psi}$ ).

The 3.8 liter plenum was then charged to a higher pressure with $\mathrm{N}_{2}$. The use of a 3.8 times larger mixing chamber compared to a prior Orion Crew Exploration Vehicle (CEV) RCS jet test ${ }^{14}$ produced a more constant NO composition during the runs. Mixing of the $\mathrm{NO}$ and $\mathrm{N}_{2}$ was enhanced by flowing the input gas into the plenum through a cylindrical tube having dozens of holes around the circumference and down the 10.4-in length of the tube. A feedback-controlled pressure regulator was used to set the jet's operating pressure, but the primary flow rate of the RCS jet was determined by a 0.030 -in diameter choked orifice plate which was placed upstream of the NO plenum. The jet's plenum (chamber) pressure inside the model, $\mathrm{P}_{\mathrm{c}}$, was between 0 to $+7 \%$ of the target pressure on most runs. In each case, the pressure was measured to $\pm 1 \mathrm{psi}$ and is indicated in the relevant figure captions reported in this study. However, when the jet was turned on in the middle of the run or when $\mathrm{P}_{\mathrm{c}}$ was changed during a run, the pressure took tens of seconds to stabilize. These pressures could differ from the set pressure by as much as $12 \%$. The 
regulator set pressure was maintained higher than the desired jet chamber pressure because of pressure drop across the sonic orifice and also pressure drops in the tubing between the orifice and the model. These pressure drops were determined experimentally prior to the wind tunnel tests. All runs had at least part of the run with a set chamber pressure, $\mathrm{P}_{\mathrm{c}}=122 \mathrm{psi}$. For this condition, the mixing chamber was pressurized above $122 \mathrm{psi}$ with the pure $\mathrm{N}_{2}$ prior to the run, thereby diluting the NO by a factor of 20 , decreasing the NO concentration to $5 \%$. Once the test began, this gas flowed into the model while more pure $\mathrm{N}_{2}$ flowed into the cell to maintain the operating pressure. A drawback of this system was that the NO concentration still decreased somewhat during the runs, though conditions did not change noticeably over a 10 -second period (the typical time required for runs in which the laser sheet is scanned through the jet). In some runs, the RCS jet did not operate. Instead, pure NO was seeded into the flow through a gap between the sting and the shroud placed over the sting. This NO was entrained in the separated wake flow behind the capsule, visualizing the shape and size of the separated wake region. For other runs, both the RCS jet was seeded with NO and the wake flow was also seeded with pure NO. Results were insensitive to the wake seeding flow rate over the range of $30-1000 \mathrm{sccm}$. This indicates that possible artificial heating effects from seeding pure NO and the possible chemical decomposition of the NO were negligible.

\section{PLIF Visualization and Velocity Measurement Techniques}

The NO PLIF system uses an ultraviolet laser sheet to interrogate a slice in the flow containing seeded NO. This UV light excites fluorescence from the NO molecules, which is detected by a high-speed digital camera. Details of the measurement system used in this experiment, as well as the experimental procedure used, can be found in References 14, 17 and 18. The laser system uses an Nd:YAG laser to pump a Sirah Cobra Stretch dye laser and Sirah Frequency Conversion Unit (FCU). The dye laser was operated near $624 \mathrm{~nm}$ and was sum-frequency mixed with the third harmonic of the Nd:YAG laser to produce the UV light used to excite NO. The resulting laser output, at $226.256 \mathrm{~nm}$, was tuned to excite the strongly fluorescing spectral lines of NO near the $\mathrm{Q}_{1}$ branch band head (where $\mathrm{Q}$ denotes a change in rotational quantum number equal to zero). Optics formed the resulting beam into a laser sheet that was $110 \mathrm{~mm}$ wide and $<1 \mathrm{~mm}$ thick (FWHM) in the measurement region. Approximately $1 \mathrm{~mJ}$ of laser energy per pulse was delivered into the test section over a duration of $10 \mathrm{~ns}$.

\section{A. Flow Visualization}

For the flow visualization data presented in this paper, the same cameras, excitation schemes, etc., were used as in past work, ${ }^{14}$ except that higher efficiency filters (Layertec $\mathrm{GmbH}$, Germany, <1\% transmission at $226 \mathrm{~nm}$ and $>80 \%$ transmission at $235-280 \mathrm{~nm}$ ) were used to transmit the LIF signal while rejecting the laser scatter. The fluorescence was imaged onto a gated, intensified $\mathrm{CCD}$ at a viewing angle normal to the laser sheet. Images were acquired using a Cerco UV transmitting F/1.8, $45 \mathrm{~mm}$ focal length lens from EADS Sodern, yielding a spatial resolution of about 2.9 pixels $/ \mathrm{mm}$ (73.7 pixels/inch) on the model centerline and $3.1 \mathrm{pix} / \mathrm{mm}(78.1 \mathrm{pix} / \mathrm{in})$ at the location of the RCS jets A1 and B2. The images were obtained at a rate of $10 \mathrm{~Hz}$ with flow-stopping time resolution ( $<1$ microsecond).

The magnifications were measured from images acquired of a regular pattern of dots of known spacing (known as a dotcard), aligned with the plane of the laser sheet and placed at various distances from the tunnel centerline. For the flow visualization experiments, the resulting spatial resolution in the image plane was about $0.5 \mathrm{~mm}(0.02 \mathrm{in})$, assuming about 2 pixels of blurring. The camera used for the flow visualizations (Princeton Instruments Gen 2 PIMAX-2) has a 512 x 512 pixel array, with 19 micron square pixels, resulting in a field of view in the images of about $170 \mathrm{~mm}$ (6.8 inches). The sheet-forming optics were attached to a twelve-inch long translation stage which allowed the laser sheet to be scanned spatially through the flow in order to visualize different cross-sections of the flow. During such scans, the magnification of the images varies by several percent. This magnification change is corrected in subsequent image processing.

To correct for optical and perspective distortion of the images, the image of the dotcard in the test section was acquired and a corresponding undistorted image of the same dotcard was created. An image registration algorithm, UnwarpJ ${ }^{19}{ }^{\text {was }}$ then used to correct the distortion. This software is a plug-in created for the image processing software, ImageJ, a freeware image processing program available from National Institutes of Health. ${ }^{20}$ To enhance the flow features in the images, the images were given a false color table with arbitrary scaling. Furthermore, a twopercent threshold has been applied to images used in the volumetric renderings. In this case, all counts below $2 \%$ of the maximum were set to zero. 


\section{B. Velocity Measurement}

A streamwise velocity measurement, detailed in Section V.D, was performed in the wake of the flow with the B2 jet pair activated. The velocity magnitude was determined by measuring the displacement of a series of fluorescence-tagged lines that were aligned vertically in a plane oriented parallel to, and offset $-39 \mathrm{~mm}$ from, the model's plane of symmetry (i.e. $y=0 \mathrm{~mm}$ ). The lines were formed by placing a 50-mm-long, anti-reflection coated LaserOptik $\mathrm{GmbH}$ diffusion welded lens array between the test section and sheet-forming optics. The lens array consisted of 25, 1-m focal length cylindrical lenses diffusion welded together that focused the laser sheet into a series of 25 vertical, parallel lines, spaced approximately $2.6 \mathrm{~mm}$ apart. Each laser line subsequently excited fluorescence in NO, which was seeded into the wake and RCS jet gases. A Cooke DiCAM-PRO camera, utilizing an intensified 1280x1024 pixel array interline progressive scan CCD, was used to image the tagged lines. The camera was capable of acquiring two sequential frames (referred to as the $1^{\text {st }}$ and $2^{\text {nd }}$ exposure) when operated in double shutter mode, separated by approximately $560 \mathrm{~ns}$ in time. Details of how this separation time is calculated can be found in the literature. ${ }^{21,22} \mathrm{~A}$ systematic comparison of the frame pairs was used to determine the displacement of each tagged line. The timing sequence used for the velocity measurement was similar to that used in Ref. 23, with the effective $1^{\text {st }}$ exposure and $2^{\text {nd }}$ exposure being $15 \mathrm{~ns}$ and $100 \mathrm{~ns}$ in duration, respectively. As with the flow visualization experiment, a Layertec $\mathrm{GmbH}$ filter $(<1 \%$ transmission at $226 \mathrm{~nm}$ and $>80 \%$ transmission at 235-280 $\mathrm{nm}$ ) was used to block the laser scatter occurring along the model surface and pass the fluorescence emitted from the tagged lines. The camera was fitted with a $100-\mathrm{mm}$ focal length, F/2.0 B. Halle Nachfl. lens, yielding a spatial resolution of approximately 15.6 pixels/mm (396.2 pixels/inch) at the measurement plane.

The initial images obtained from the velocity measurement experiment were hardware binned by 2 pixels in the vertical direction to increase the frame rate by a factor of two. Background images were created by separately averaging a series of images from the $1^{\text {st }}$ and $2^{\text {nd }}$ exposures while the laser was blocked, which were then subtracted from the initial velocity images. Due to the shortness of the duration between sequential images (500 ns) and the relatively long decay time of the P46 phosphor in the intensifier, some ghosting remained from the $1^{\text {st }}$ exposure in the $2^{\text {nd }}$ exposure. For this $500 \mathrm{~ns}$ delay, the ghosting level was experimentally estimated to be approximately $1.2 \%$ of the signal in the $1^{\text {st }}$ exposure. This level was also subtracted from the $2^{\text {nd }}$ exposure images.

The velocity was determined for each point along the tagged profiles by using a 1-dimensional cross-correlation technique between windowed horizontal regions in each $1^{\text {st }}$ and $2^{\text {nd }}$ exposure image pair. For each single-shot data point, a coarse velocity estimate was first obtained from windowed regions that were 45-pixels wide and were staggered vertically in 10-pixel increments. Once a coarse estimate was made, the windows were reduced to a width of 15-pixels, the $2^{\text {nd }}$ exposure window was shifted horizontally based upon the coarse velocity estimate, and a refined velocity estimate was made. For each data point, the single-shot images from which the windowed regions were obtained were smoothed with a 2-pixel radius disk filter and were further binned together in order to improve signal-to-noise levels. However, this meant that there was a possibility of incurring inaccuracies in the velocity measurement due to signal saturation and phosphor artifact effects. To avoid this, first a threshold of 3000 counts was chosen and any horizontal rows within the single-shot image pairs having counts exceeding this threshold were removed. The subsequent single-shot images were then smoothed with the 2-pixel radius disk filter and the region that corresponded to the original windowed area was binned vertically and a velocity value obtained. This process was repeated on a point-by-point and shot-by-shot basis.

The resulting spatial resolution of each velocity data point was approximately $1.28 \mathrm{~mm}$ in the vertical direction and $2.6 \mathrm{~mm}$ in the horizontal direction. The DiCAM Pro has a 1280x1024 pixel array, resulting in a field of view of approximately $82 \mathrm{~mm}$ (3.2 inches) in the vertical direction and $66 \mathrm{~mm}$ (2.6 inches) in the horizontal direction. Similar to the procedure described in the processing of the flow visualization images, the velocity measurement images have also been corrected for perspective and lens distortions. ${ }^{18}$

\section{ViDI Processing}

As mentioned above, the ViDI method was used to process the image data and to generate renderings that bring out the three-dimensional nature of the flow visualization data while showing the relative location of the data with respect to the model. ${ }^{18}$ Most of the runs were processed in a consistent manner with Autodesk ${ }^{\circledR} 3 \mathrm{ds}$ Max ${ }^{\circledR}$ software with customized scripts. The placement of the images with respect to the model is estimated to be accurate within 1 $\mathrm{mm}$ (0.04 inches). However, one anomaly was observed that was inconsistent from run-to-run and was attributed to the movement of the MSL model with respect to the sting. The spatially-corrected PLIF images showed that the model did not always align perfectly with the reference marks in the images (i.e. surface scattered light and/or shadows caused by the model). These apparent movements of the model may be due to aerodynamic loading or thermal effects on the model. Alternately they could be caused by slight drift of the camera system during the runs. 
To correct for this misalignment, the images were aligned within the software to the sting, which remained fixed, while the capsule model was rotated (less than a degree) and translated (less than 0.04 in) such that the image markings were consistent with their relative positions with respect to the virtual model. The degree to which the model had to be rotated and translated within the software typically depended on the angle of attack, which changed within a single run. Nonetheless, theses effects are small and do not impact the overall results.

\section{Results}

Through NO PLIF flow visualization, the influence of model AoA on flow separation and the structure of the three dimensional wake behind the MSL vehicle were studied. In addition to the wake-flow, both jet-crossflow interactions and jet-jet interactions were studied through visualization of the RCS jets directly. Molecular tagging velocimetry was used to gain quantitative measurements of the streamwise component of velocity in the MSL wake. This benchmark data is intended to be used for numerical model validation of hypersonic computational fluid dynamic simulations.

\section{A. Wake Flow Visualization without RCS Jets}

Figure 6 shows NO fluorescence images obtained using a wake-seeding technique, which aids in the visualization of the near-wake structure behind the MSL model for a typical tunnel test. These tests are referred to as jet-off cases, where the RCS jets were not activated. The NO seeding flow rate was varied to demonstrate the effects that the seeding gas had on the structure of the flow field. The laser sheet was positioned on the centerline of the MSL model, which was oriented at a $-20^{\circ}$ AoA. The wind tunnel stagnation pressure for this test was 1301 psi. Pure NO was seeded into the flow through a gap between the sting and the shroud placed over the sting. NO became entrained in the separated wake flow behind the capsule and was convected upstream near the separation point. For these conditions, the flow appears that it was attached around the windward heatshield shoulder junction as the freestream gas formed a $17^{\circ}$ angle with respect to the aftbody backshell. The flow separated at the backshell hip junction as the capsule surface turned a further $22^{\circ}\left(39^{\circ}\right.$ with respect to the freestream). Although it appears that the separation region was slightly downstream of the backshell hip junction at the centerline, separation is expected to have occurred at the junction. Low concentrations of NO near the separation point make it difficult to determine the exact separation location. As shown in the comparison between Fig. 6a and 6b, NO seeding flow rates over the range of $30 \mathrm{sccm}$ to $1000 \mathrm{sccm}$ result in a negligible difference in the overall flow structure, and importantly the size of the separated flow region. Results using lower seeding flow rates (i.e. Fig. 6b) are, however, characterized by lower signal-to-noise ratios. The PLIF signal amplitude decreased with decreasing NO concentration since the rate of fluorescence is proportional to the population of NO molecules in the ground state. For remaining wake-seeding tests, an intermediate NO flow rate of $500 \mathrm{sccm}$ was used.

(a)

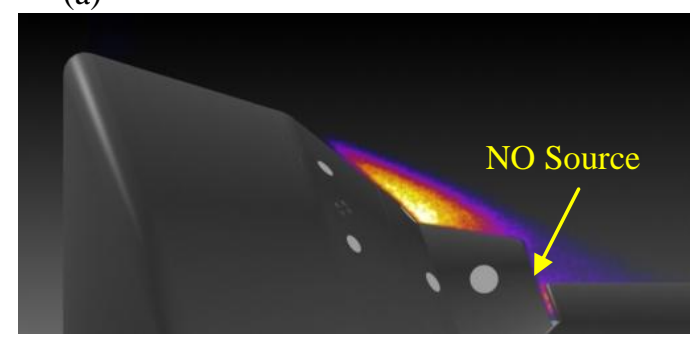

(b)

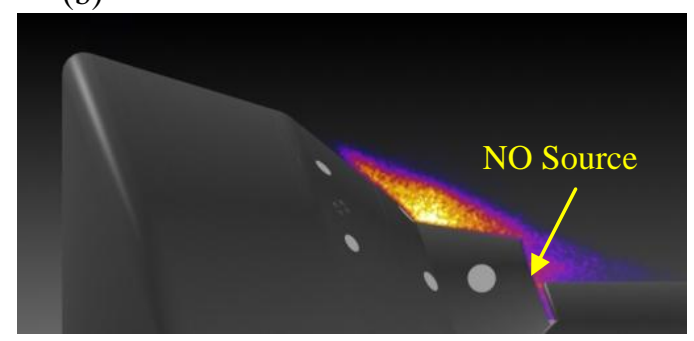

Figure 6: Effect of wake flow seeding rate. Jet off, wind on, $P_{0}=1302$ psi; a) 1000 sccm NO flow rate; b) $30 \mathrm{sccm}$ NO flow rate. Averaged PLIF Signal.

By scanning the laser sheet across the flow (Fig. 7a), a 3D separated region can be visualized as a composite of individual PLIF image slices (Fig. 7b). The overall shape of the recirculation zone is governed by the locations of separation points distributed along the aft-body backshell. Understanding the shape of the separated region is important as it dictates whether the RCS jet will interact with an attached supersonic cross-flow or with a recirculating subsonic wake-flow. Considering the model surface angle is useful to gain a basic understanding of the separated shape. The surface angle is defined as the angle that forms between the model surface and the free-stream gas flow vector. This angle is $0^{\circ}$ when the model surface is parallel to the free-stream gas. Since the model was pitched forward at a $-20^{\circ}$ AoA, the surface angle varied in the spanwise direction for a given vertical position. 
Most often the separation location occurred at either the backshell-hip junction or the heatshield-shoulder junction since sudden changes in surface angles occur at these locations. From observation, it appears that the upstream boundary of the NO PLIF signal occurred near an iso-line corresponding to a critical surface angle of approximately $26^{\circ}$. At point 1 , for example, the flow separated at the backshell hip junction, which formed surface angles over the range of $17^{\circ}$ and $39^{\circ}$. Similarly, the surface angles corresponding to the upstream and downstream surfaces near point 2 were $26^{\circ}$ and $48^{\circ}$, respectively. Between points 2 and 3, separation occurred on the backshell region between the heatshield shoulder and the backshell hip junctions near a surface angle iso-line of approximately $26^{\circ}$. Three surface angle iso-lines $\left(17^{\circ}, 22^{\circ}\right.$, and $\left.26^{\circ}\right)$ are shown in Fig. $7 \mathrm{~b}$ to aid in the interpretation. Finally, between points 3 and 4 the separation location occurred in the vicinity of the heatshield shoulder junction where the surface angle was greater than $26^{\circ}$.

(a)

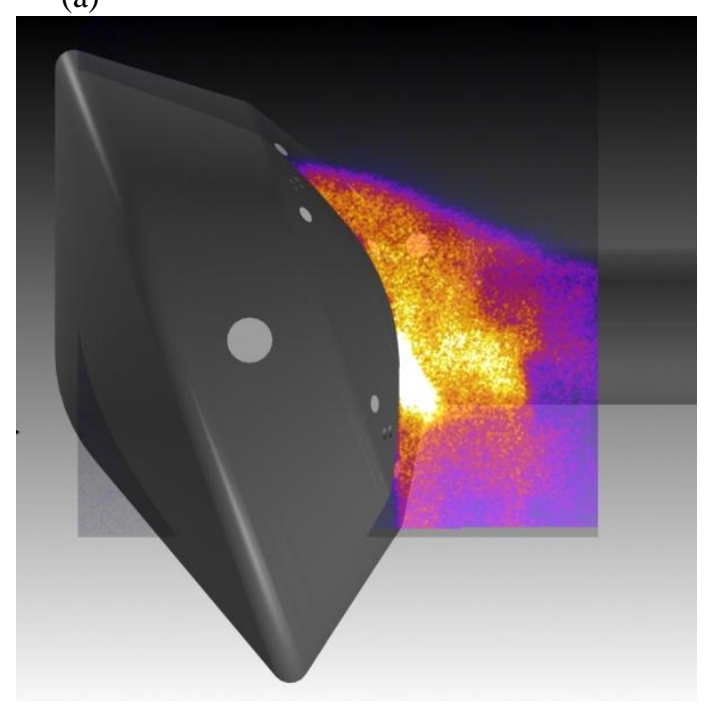

(b)

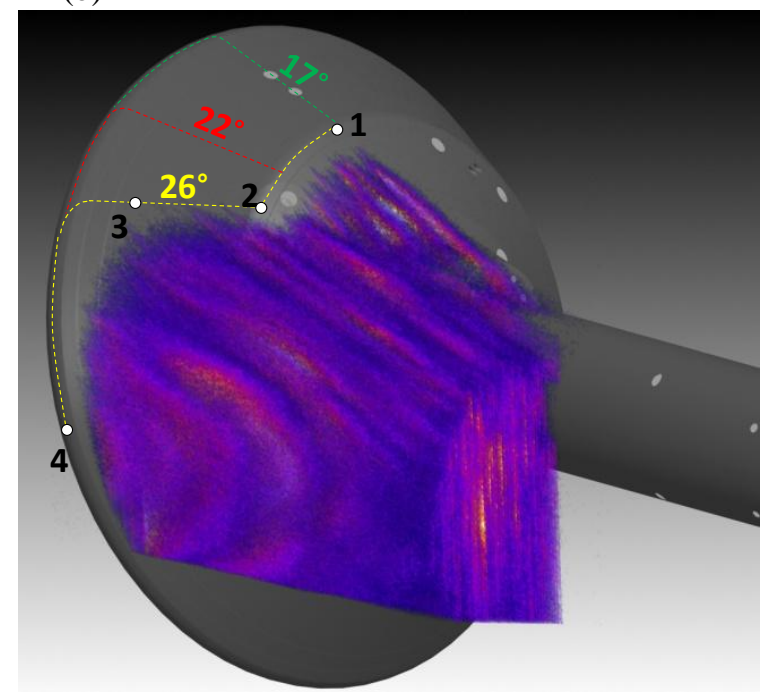

Figure 7: Wake flow PLIF visualization, jet off, $A o A=-20^{\circ}$, wind on, $P_{0}=1301$ psi; a) single shot side view; b) composite perspective view. Separation points indicated as numbers.

Figure 8 shows a comparison of average intensity and single shot intensity PLIF images over a range of model angles of attack from $\mathrm{AoA}=0^{\circ}$ to $-25^{\circ}$ in $5^{\circ}$ increments. The change of the model AoA is controlled by rotating the sting. In these images, the laser sheet was fixed at the model centerline. To assist in the comparison between images, the frame of reference is fixed on the model rather than being fixed on the wind tunnel. At $-25^{\circ}$ AoA, the flow remained attached on the backshell surface after the heatshield-shoulder and backshell-hip junctions. The only visible PLIF signal is at the end of the PCC where the NO is injected into the wakeflow. It is possible that separation occurred at either of the two previous junctions, but then reattached before reaching the NO source. The PLIF images indicate that the flow separated at backshell-hip junction at $-20^{\circ}$ AoA. The intensity of the single-shot images at this condition is very sporadic from frame-to-frame. This suggests that occasional reattachment on the PCC occurred, which temporarily inhibited NO from accumulating in the separated region behind the backshell-hip junction. In all of the single-shot images, no PLIF signal is observed upstream of the backshell-hip junction. At $-15^{\circ}$ AoA, the average intensity images show a much larger separated region originating from the backshell-hip junction, extending past the PCC, and reattaching on the sting outside the laser sheet field of view. The single shot images show that the point of separation is unstable and occasionally moves upstream past the backshell junction causing a dark band of low luminescence to form in the image. It appears that $-15^{\circ} \mathrm{AoA}$ is near the critical angle required to move the transition point upstream of the backshell hip junction. For model angles of attack less than $-10^{\circ}$ the separation point occurs at the heatshield-shoulder junction. At $-10^{\circ}$ AoA, the surface angle that forms between the freestream gas and model backshell is $27^{\circ}$, which is similar to observations made above regarding the 3D separated region shown in Fig. 7. At AoAs of $-5^{\circ}$ and above, the model extends out of the field of view of the laser sheet. Since the expansion around the heatshield shoulder junction was amplified at higher AoA, it is expected that the separation point remained at the heatshield shoulder junction at the higher AoAs. 

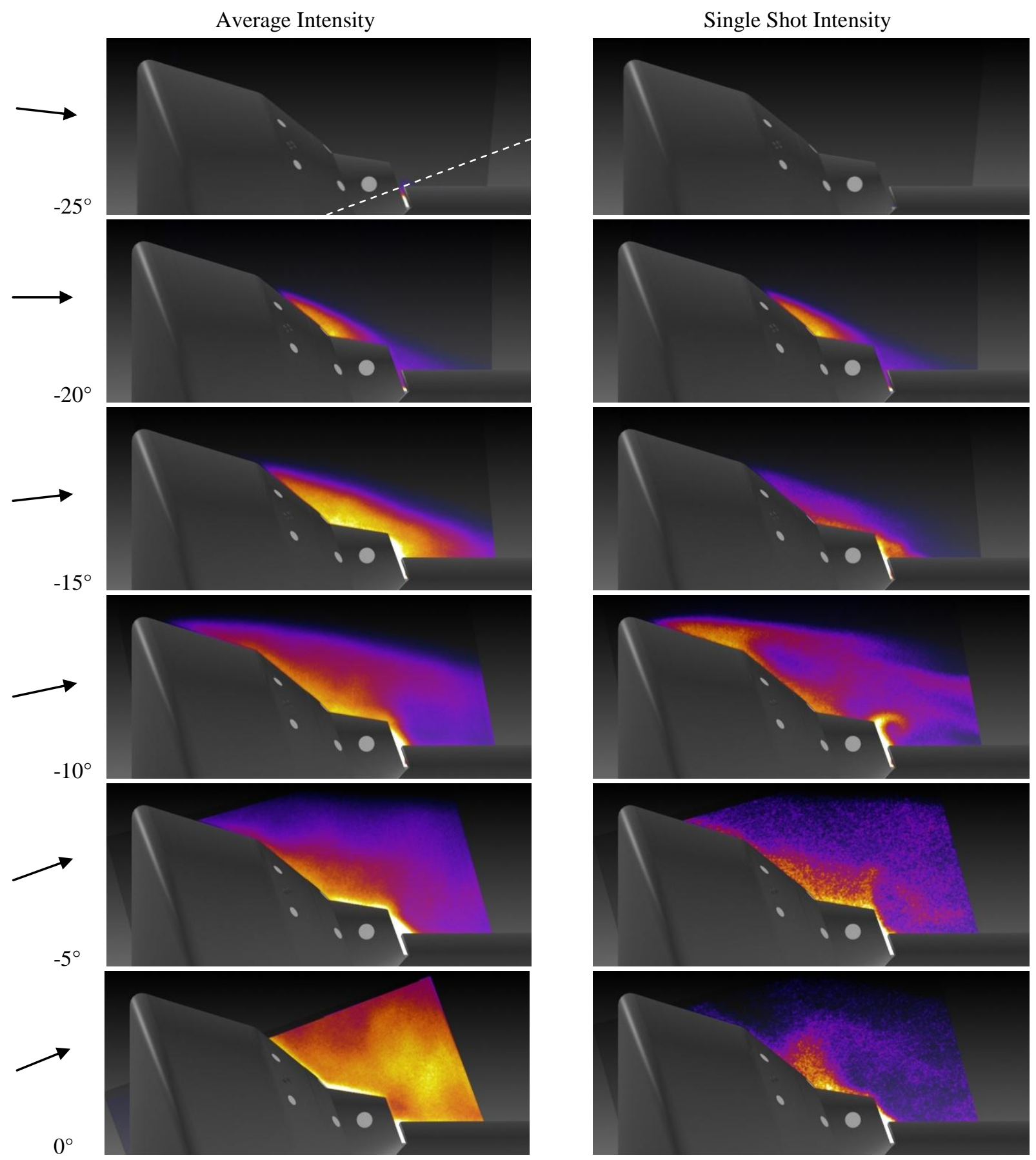

Figure 8: Effect of AoA on wake PLIF visualization. Left column is average PLIF signal and right column is single-shot PLIF signal. Jet off, wind on, $P_{0}=1302$ psi. Model frame of reference. Arrows show direction of freestream gas. White dashed line shows center axis of model. Images have been scaled arbitrarily. 


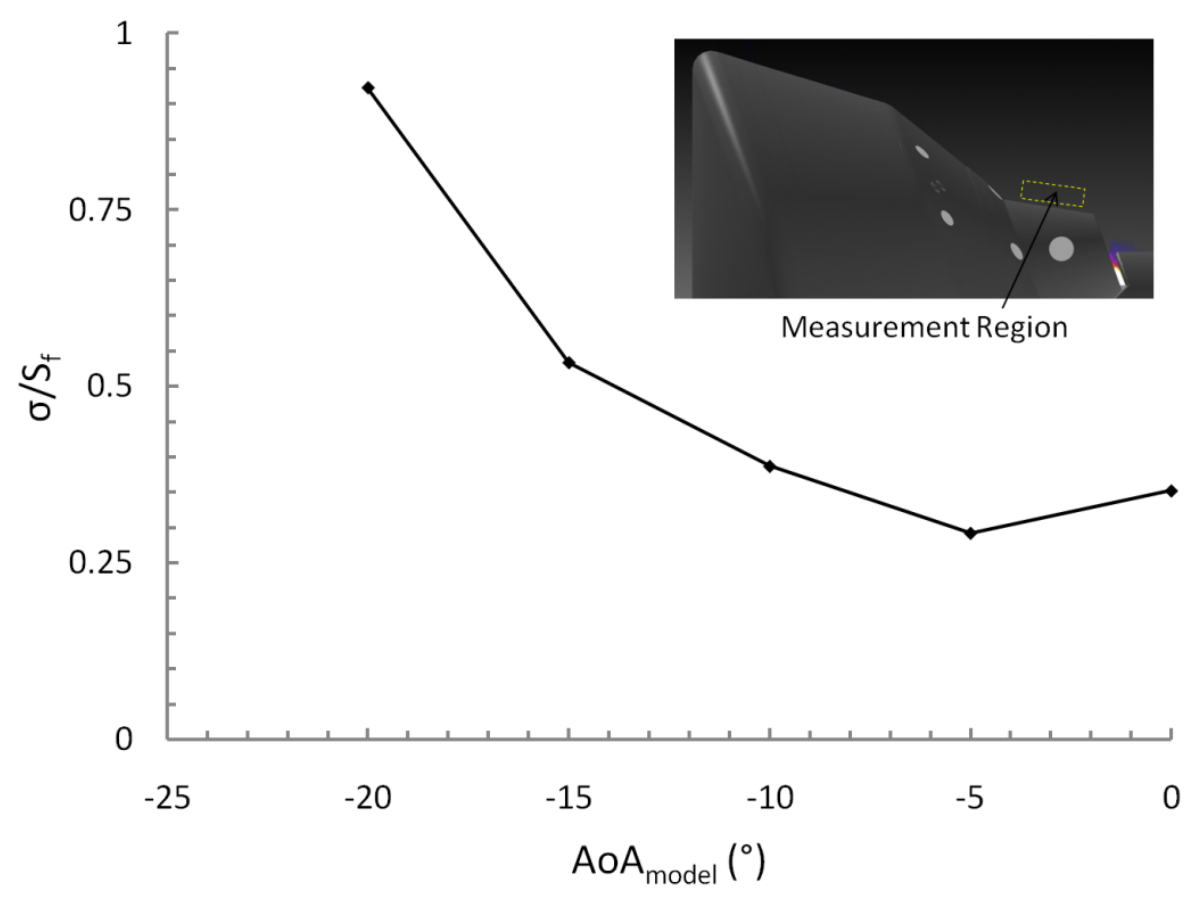

Figure 9: Fluctuations in the PLIF signal $\left(\sigma / \mathrm{S}_{\mathrm{f}}\right)$ measured in the recirculation zone above PCC. $\mathrm{S}_{\mathrm{f}}$ is the mean signal in measurement region shown in figure.

The sporadic frame-to-frame signal intensity observed at high AoA is quantified in Figure 9. In the figure, the standard deviation in signal intensity is normalized by mean signal values for a range of model AoA. The measurement region indicated in the inset figure corresponds to a location above the PCC. At $-20^{\circ}$ AoA the standard deviation of signal intensity approaches the same order of magnitude as the signal mean. This confirms intermittency of the fluorescence signal observed in the single shot images at this condition. As the magnitude of AoA decreases (i.e. $|\mathrm{AoA}|<20^{\circ}$ ), the ratio of standard deviation to mean signal levels also decrease indicating that the seeded NO gas was able to convect continuously into the separated regions created upstream on the backshell surface. At AoA $=-25^{\circ}$, however, NO was unable to convect from the sting source location forward to the PCC, which led to very small magnitudes of both signal standard deviation and signal mean levels.

As the model AoA changes, sudden changes in the flow separation position is accompanied by redistributions in the surface pressure distribution. Although aerodynamic forces and moments are dominated by the surface pressure distribution on the model forebody in hypersonic flows, subtle changes in these quantities can be attributed to the structure of the wake-flow. Figure 10 shows the effect of model AoA on the pitching moment coefficient, $C_{m}$, which is defined as the aerodynamic pitching moment $(M)$ normalized by the dynamic freestream pressure $(q)$, capsule area $\left(A_{H S J}\right)$, and capsule diameter $\left(D_{H S J}\right)$. From $0>$ AoA $>-10^{\circ}, C_{m}$ varies linearly with AoA, which is driven by the forebody pressure forces. Between $-10^{\circ}$ and $-15^{\circ}$, however, there is a change in the slope of $C_{m}$, which coincides with a change is separation location from the heatshield shoulder junction to the backshell hip junction. The transition is smooth due to the three dimensionality of the capsule surface as the local separation angle between the model surface and freestream gas changes with spatial location. A second, more subtle transition in $C_{m}$ occurs at approximately $-25^{\circ}$ AoA, where the separation location moves from the backshell hip junction to the aft-side of the PCC. 


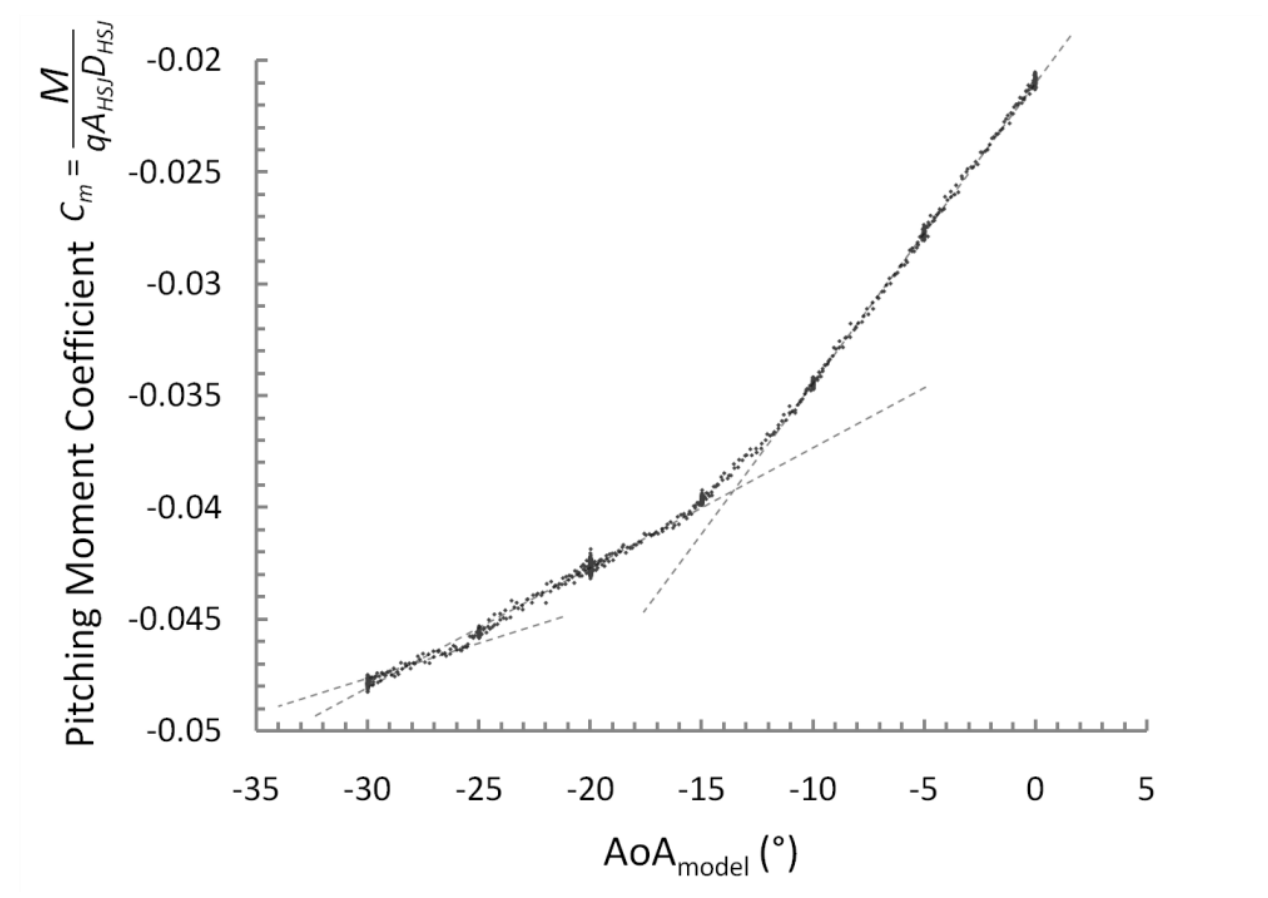

Figure 10: Model pitching moment coefficient $\left(C_{m}\right)$ vs AoA. Dashed lines overlaid to highlight linear regions. $M$ is the model pitching moment, $A_{H S J}$ is the area of the capsule, $q$ is the freestream dynamic pressure, and $D_{H S J}$ is the diameter of the capsule.

Flow separation is influenced by many factors in addition to the local surface angle, including the location of the rear stagnation point, capsule base pressure, and turbulence. ${ }^{8,24}$ Empirical correlations relating the separation angle to the post-shock Reynolds number were developed by Park et al. through the investigation of a laminar near-wake region behind a circular cylinder in a hypersonic flow. ${ }^{25}$ The correlation was compared to experiments from wind tunnel and shock tunnel facilities over the Mach number range of 6 to 14. The correlation adapted to the coordinate system used in this study is defined as:

$$
\theta_{\text {sep }}=26.3+577 \mathrm{Re}^{-1 / 2}
$$

The separation angle originally defined by Park et al. is the angular position of the separation point relative to the upstream stagnation point on the cylinder. For example, separation occurring at the top of the cylinder would correspond to an angular position of $90^{\circ}$. For circular cylinders, an angular position of $90^{\circ}$ corresponds to a surface angle of $0^{\circ}$ with respect to the free-stream flow vector. Since the MSL capsule is not cylindrical, it is not useful to describe the separation location in terms of an angular position. Instead, it is useful to report the surface angle, which is why the correlation in Eqn. 1 was adapted. Note that in Ref. 25, the slope and intercept were inadvertently transposed. ${ }^{26}$ This has been corrected in Eqn. 1.

Based on post-shock flow conditions and the MSL capsule diameter, Eqn. 1 can be applied to predict the critical separation angle for the MSL model. With a Reynolds number of approximately $8\left(10^{4}\right)$, the critical separation angle is approximately $\theta_{\text {sep }}=28^{\circ}$, which is very similar to observations of the MSL 3D separated flow (Fig 7b, $\theta_{\text {sep }}=26^{\circ}$ ) and to observations of separation along the model centerline (Fig 8, AoA $=-10^{\circ}, \theta_{\text {sep }}=27^{\circ}$ ). Although the MSL vehicle is not cylindrical in shape, this simplified correlation is useful in determining a basic overall separation shape and to help predict the influence of model vehicle angle of attack on separation. Discussion will now turn to the interaction between the RCS jets and the MSL wake-flow.

\section{B. Jet-Flow Interaction}

To understand the effects of the MSL wake-flow on the RCS jet structure, the RCS jets were operated in both wind-off and wind-on tunnel conditions. The wind-on condition refers to tests where the wind tunnel was used in a normal operating mode, providing a Mach 10 flow into the test section. During the wind-off condition, the wind 
tunnel was not operated, allowing RCS jets to eject fluid into quiescent surroundings. The static pressure in the test section, however, was maintained at a similar wake static pressure associated with the wind-on case. Unfortunately, the vacuum spheres used to maintain low pressures were not able to perfectly match the low wake pressures that occur naturally from flow expansion during tunnel operation. As a result of a higher ambient pressure, the jet spreading rate was artificially suppressed in the wind-off case. Figure 11 shows a comparison between the wind-off and wind-on conditions with RCS jet pair A1 activated (see Fig. 1 for RCS jet locations).
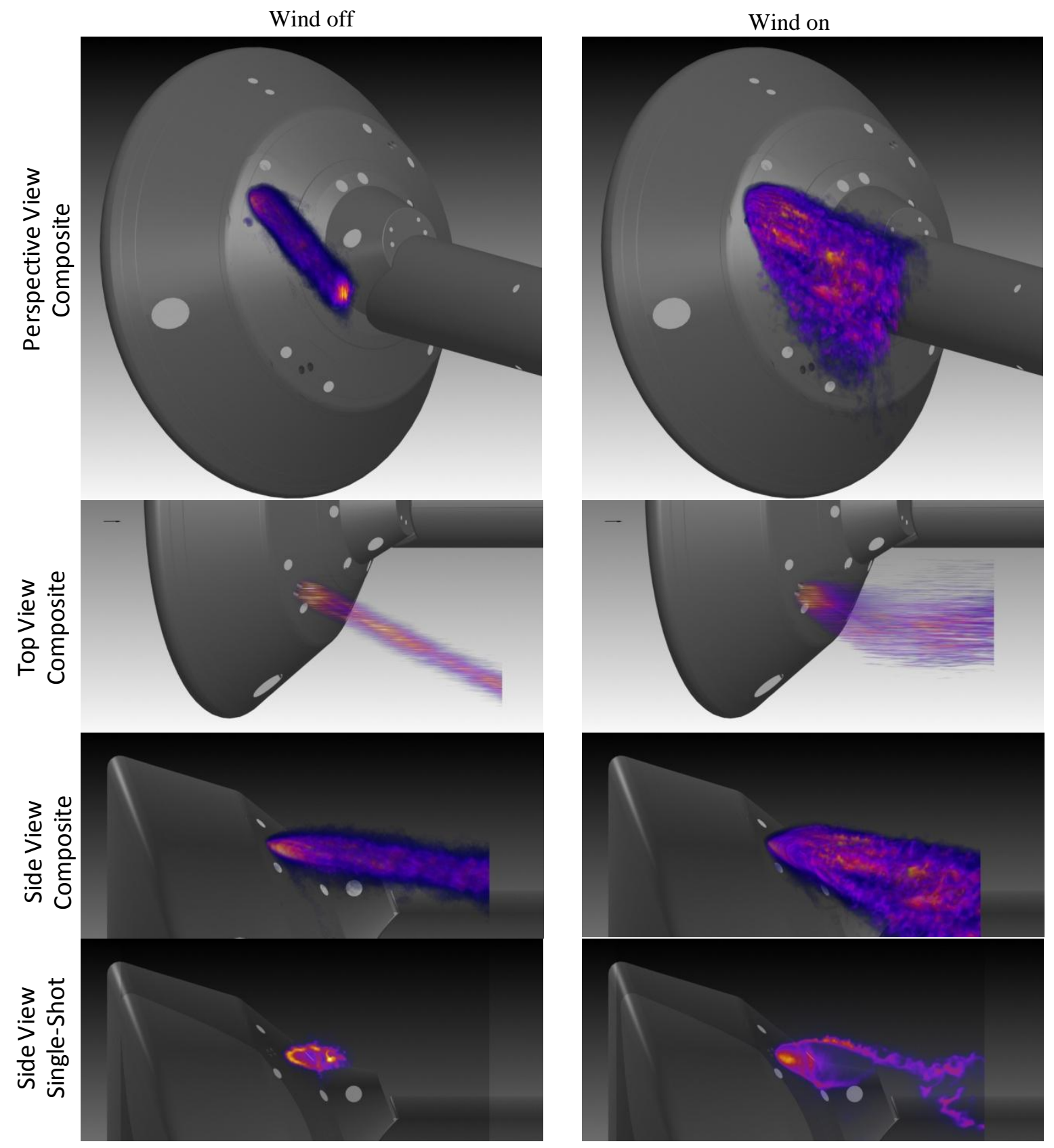

Figure 11: Effect of Jet-Flow Interaction. Jet $A 1$ activated, AoA $=-20^{\circ}$. Wind-off condition $\left(P_{c}=122 \pm 1\right.$ psi, $P_{0}=0.193$ psi). Wind-on condition $\left(P_{c}=125 \pm 1\right.$ psi, $P_{0}=1301$ psi $)$. Perspective view composite, top view composite, side view composite, and side view single-shot PLIF images are shown. 
During the wind-on condition, the tunnel stagnation pressure, jet pressure, and model AoA were $1301 \mathrm{psi}, 125 \mathrm{psi}$, and $-20^{\circ}$, respectively. The ambient pressure maintained during the wind-off case was approximately four times higher than the wake static pressures measured in the wind-on case. The top three rows in the figure show perspective, top and side view volume renderings of the data measured during the laser-sheet sweeps through the flow. The bottom row shows single-shot images with the laser sheet located near the nozzle exit. The three dimensionality of the flow along with small turbulent flow structures are observed in both wind-off and wind-on cases.

As expected, the jet trajectory relative to the model centerline for the wind-off case was unaffected with downstream distance due to an absence of a crossflow. In contrast, the interaction of the RCS jet with the crossflow in the wind-on case deflected the jet downstream and back towards the sting. From observations of the separated flow structure shown in Fig. 7, RCS Jet A1 is expected to be initially shrouded in a subsonic re-circulating flow when the model AoA is at $-20^{\circ}$. As a result, a bow shock is not expected to have formed upstream of the jet nozzle exit at this condition as depicted in Fig. 4a. Barrel shock waves, however, are expected to have formed at the nozzle exits for both the wind-on and wind-off cases. The location of a shock wave is coincident with sudden changes in the PLIF signal. A sudden increase in the gas density amplifies the PLIF signal, while an increase in the static pressure suppresses the PLIF signal through collisional quenching. In the wind-on condition, a large lee-side shear layer forms upstream of the barrel shock wave. The associated large-scale lee-side vortices that form are clearly visible in the single-shot image in Fig. 11. The long shear layer, showing the development of Kelvin-Helmholtz instabilities, is visible in the images because the jet trajectory becomes aligned with the laser sheet. In contrast, the RCS jet maintains its initial trajectory in the wind-off case and only a small oval-shaped diagonal slice is extracted in the single-shot images.

\section{Jet-Jet Interaction}

Simultaneous activation of both A1 and B2 jet pairs results in a jet-jet interaction in addition to the jet-crossflow interaction that was discussed above. Figure 12 shows a wind-on vs. wind-off comparison from tests with both A1 and $\mathrm{B} 2$ jets activated. For the wind-off condition, the ambient pressure was lower $\left(\mathrm{P}_{0}=0.078 \mathrm{psi}\right)$ than the ambient pressure shown in the wind-off case in Fig. $11\left(\mathrm{P}_{0}=0.193\right.$ psi).
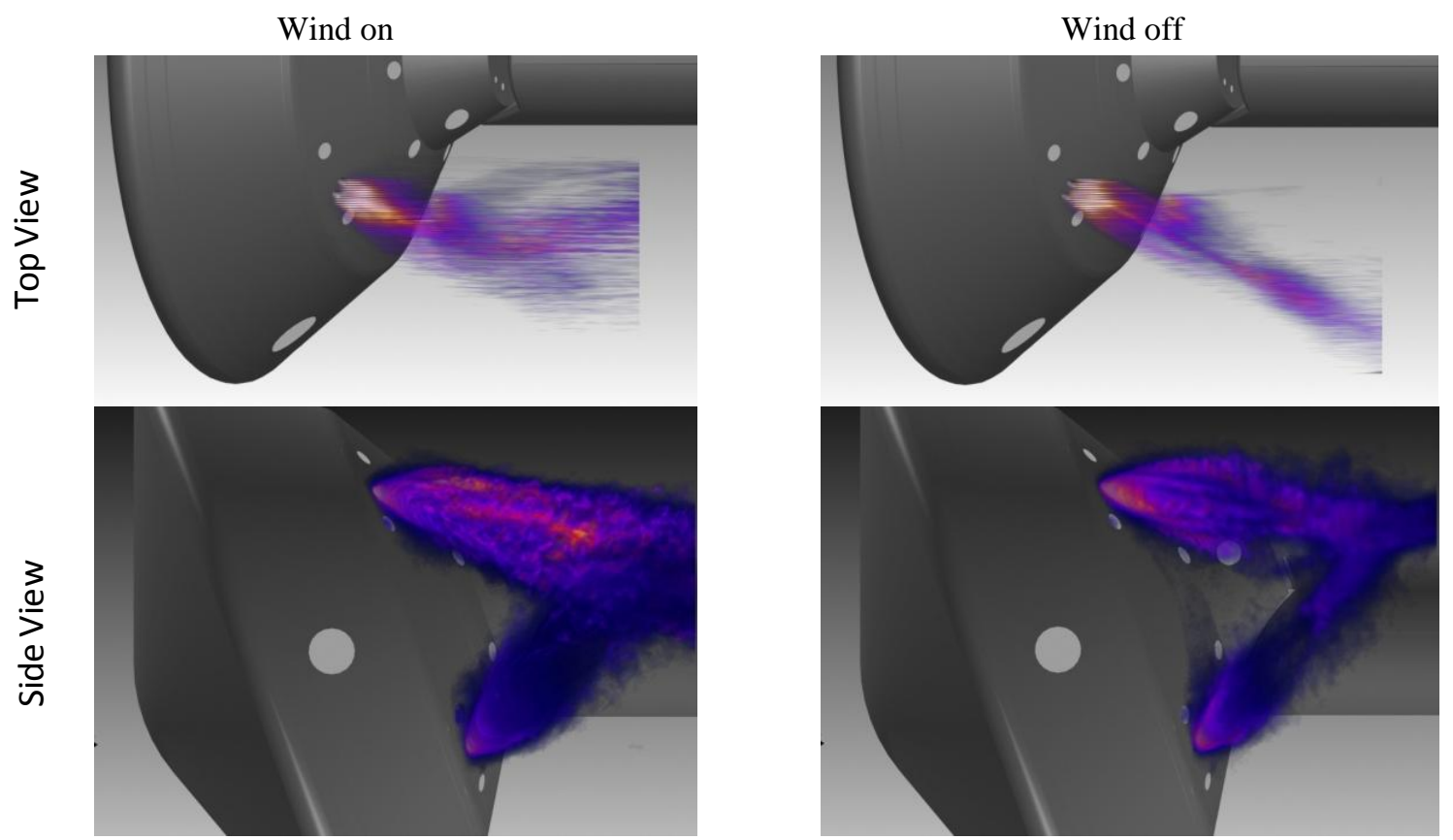

Figure 12: The effect of crossflow on RCS jet-jet interaction. Jets A1\&B2; AoA $=-20^{\circ}$; Wind-on $\left(P_{c}=\right.$ $\left.127 \pm 0.5 \mathrm{psi}, \mathrm{P}_{0}=1302 \mathrm{psi}\right)$; Wind-off $\left(P_{c}=126 \pm 1 \mathrm{psi}, \mathrm{P}_{0}=0.078 \mathrm{psi}\right)$; top-view and side-view composites of single-shot images shown. 
As a result the spreading rate of the jet (wind-off) was larger and more closely matched the jet structure for the windon case. Comparison between the top and side views reveals that the crossflow similarly affects the trajectory of A1 and B2 jet pairs, deflecting them both towards the sting. This change in trajectory results in a shorter merging distance between the two jet pairs for the wind-on cases compared to the wind-off cases. Although the distance is shorter, the images do not indicate a significant jet interaction that will alter the moments acting on the model.
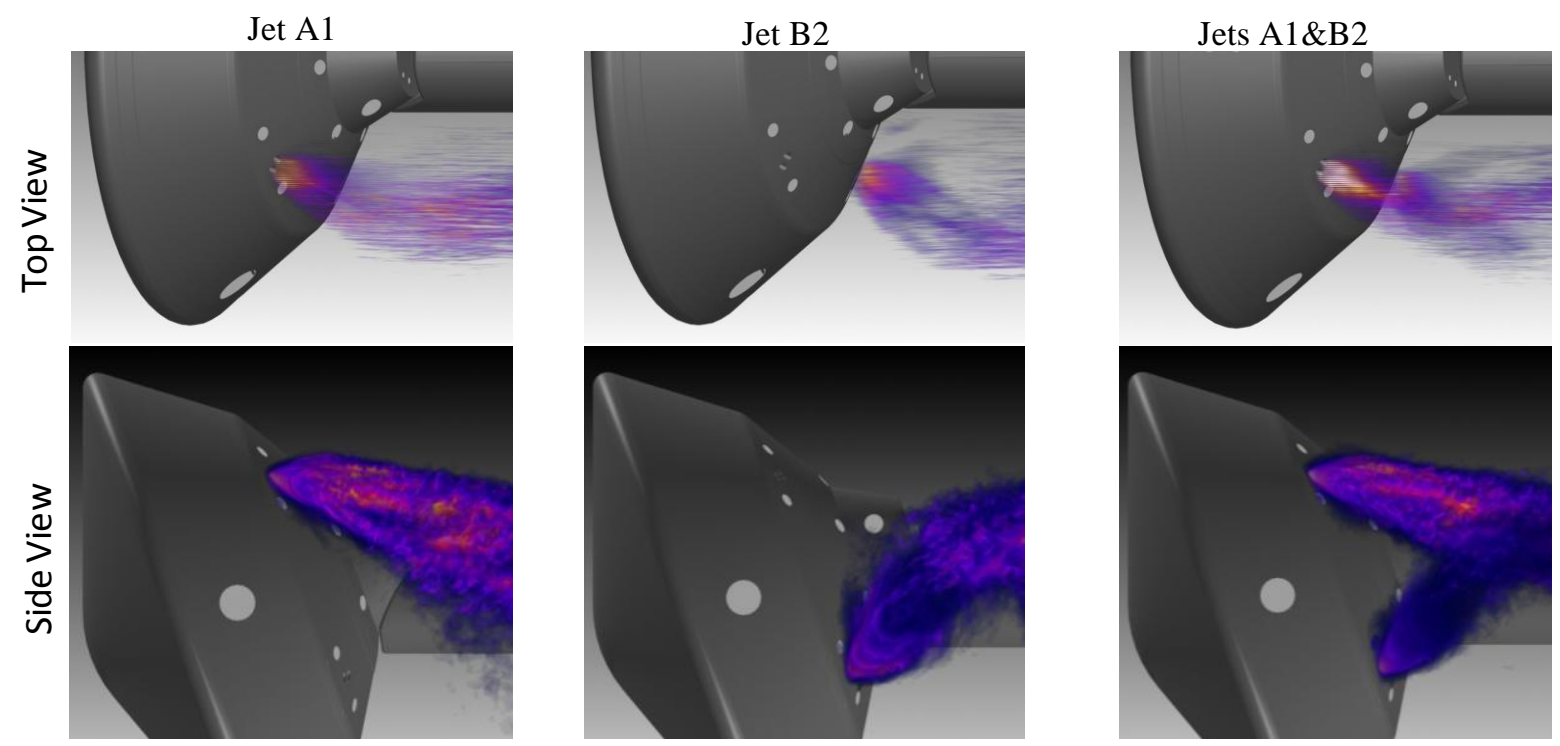

Figure 13: RCS jet interaction. Jet A1 $\left(P_{c}=125 \pm 1\right.$ psi, $P_{0}=1301$ psi $)$; Jet A2 $\left(P_{c}=126 \pm 1\right.$ psi, $P_{0}=1302$ psi); Jets A1\&B2 $\left(P_{c}=127 \pm 0.5 \mathrm{psi}, P_{0}=1302 \mathrm{psi}\right) . \mathrm{AoA}=-20^{\circ}$; top-view and side-view composites of single-shot images shown.

Figure 13 shows wind-on results from tests with individual jet pairs A1 or B2 activated compared to tests with both A1 and B2 jet pairs activated. Comparison between the top and side views reveals that the crossflow similarly affects the trajectory of A1 and B2 jet pairs regardless if they are activated individually or in combination. It does appear, however, that the B2 jets partially inhibit the expansion of the A1 jets. Although subtle, A1 jets are observed to propagate further to the bottom right of Fig. 13 when fired individually compared to when fired in combination with B2 jets.

\section{Molecular Tagging Velocimetry}

NO PLIF molecular tagging velocimetry ${ }^{21,22}$ was used to characterize the wake flow with the B2 RCS jet pair activated. NO was seeded both into the B2 RCS jets and from the junction between the model and sting. A lens array was placed between the sheet-forming optics and the measurement region to generate a pattern of lines in the image. Figure 14 shows the integrated signal of 62 such images for a typical run with and without the RCS jets activated. The lens array focuses the portions of the laser sheet into a series of discrete collimated beams, which are visible in the figure. When the RCS jets were activated, NO concentration in the jet was much higher than in the wake. As a result, the PLIF signal in the jet overwhelmed the PLIF signal in the surrounding gas. Consequently, in Fig. 14 different arbitrary scalings have been applied to the images during rendering in ViDI. In some cases the PLIF signal was too large and exceeded the dynamic range of the camera. When the RCS jets were not activated, however, a more uniform distribution of NO existed in the wake. The vertical extent of the MTV lines can be seen in the left image of Fig. 14. The PLIF signal rapidly decreases in magnitude near the shear layer as the NO concentration diminishes from dilution with the unseeded crossflow. Using a separate camera system, images were acquired in pairs, separated by $560 \mathrm{~ns}$. As the excited NO molecules were convected with the free-stream flow, a shift in each line was observed between the $1^{\text {st }}$ and $2^{\text {nd }}$ exposure images. The laser sheet traversed a set of six equally spaced spanwise distances, stopping at each location for 6 seconds to allow 60 images to be obtained at each location for statistical analysis. Through post-processing, it is possible to measure quantitative distributions of streamwise velocity at each of the line locations. In addition, to further understanding the flow field near the RCS jets, such information is also useful as a benchmark dataset for CFD comparison. 
Jet Off

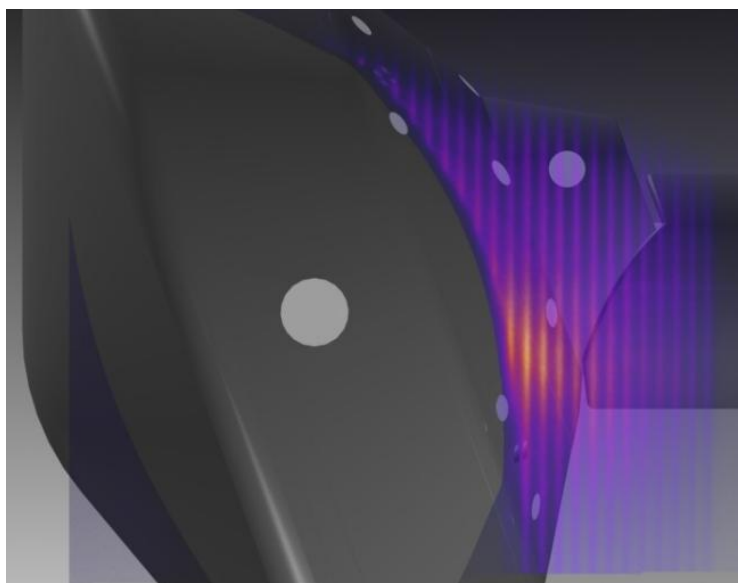

Jet B2

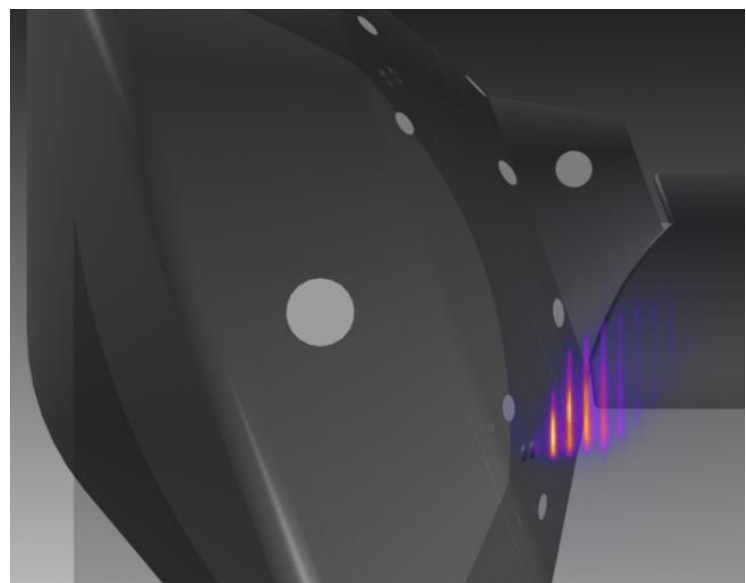

Figure 14: Sample image of illuminated lines of NO used for molecular tagging velocimetry. Jet Off $\left(P_{0}=1301\right.$ psi). Jet $B 2\left(P_{c}=128 \pm 2 \mathrm{psi}, P_{0}=1301 \mathrm{psi}\right)$. AoA $=-20^{\circ}$; side view shown; Laser sheet at $y=-39 \mathrm{~mm}(-1.53 \mathrm{in})$. The left image was processed with a much higher arbitrary intensity scaling than the right.

At each plane, a contour map of streamwise velocity can be created through the interpolation of each of the MTV lines onto a two dimensional plane using the software, Tecplot ${ }^{\circledR}$. A sample of one of the contour maps of streamwise velocity $\left(V_{x}\right)$ is shown in Figure 15. In this figure, the laser sheet is positioned at $y=-39 \mathrm{~mm}$, which is the spanwise position measured from the model centerline. This spanwise position is roughly one nozzle diameter away from the RCS jet pair B2. These results correspond to a wind-on test with RCS jet B2 activated at a jet pressure of $P_{c}=128 \mathrm{psi}$. At $-20^{\circ}$ AoA, the field of view shown in Fig. 15 is located within the subsonic separated wake behind the capsule. From visual inspection of the flow visualization images shown in Fig. 7b, the streamline separating the supersonic outer-flow and the subsonic separated wake flow is expected to exist near the upper boundary of the contour map shown in Fig. 15. Data could not be obtained at higher vertical positions due to the absence of NO in and above the shear layer. From the contour map, the peak component of streamwise velocity of the RCS jet is observed to be roughly $800 \mathrm{~m} / \mathrm{s}$. The theoretical gas velocity of a fully expanded nitrogen jet with a stagnation pressure of $125 \mathrm{psi}$ and stagnation temperature of $300 \mathrm{~K}$ is approximately $800 \mathrm{~m} / \mathrm{s}$.

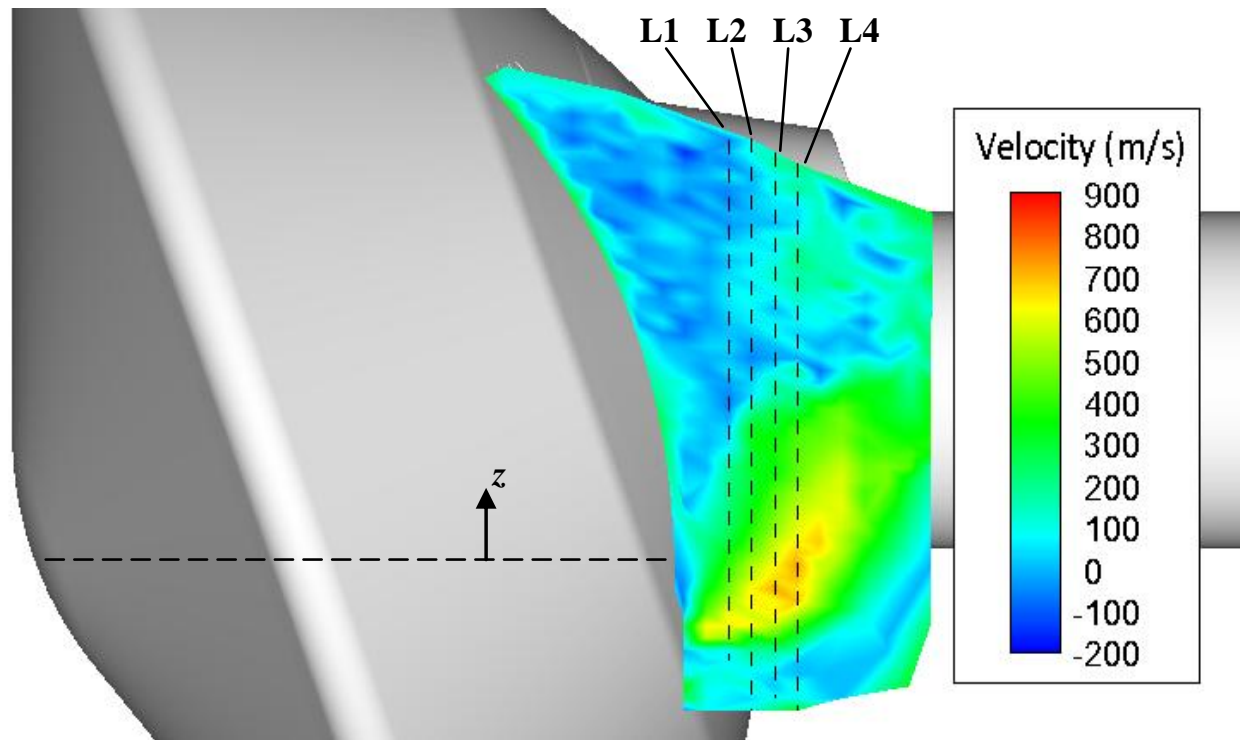

Figure 15: Contour map of streamwise velocity $\left(V_{x}\right)$. Jet $\mathrm{B} 2\left(\mathrm{P}_{\mathrm{c}}=128 \pm 2 \mathrm{psi}, \mathrm{P}_{0}=1301 \mathrm{psi}\right)$; AoA $=-20^{\circ}$; Laser sheet at $y=-39 \mathrm{~mm}(-1.53 \mathrm{in})$. 
The shape and trajectory of the jet based on the velocity measurements agrees with the qualitative images shown in Fig. 13. The four dashed vertical lines in Fig. 15 indicate four representative locations, the velocity distributions of which are shown in Fig. 16. The streamwise position of each line relative to the capsule nose is indicated in the figure. The horizontal thickness of the bars represents the uncertainty in the mean levels calculated from a statistical analysis of the individual single-shot measurements. The methodology for calculating uncertainty levels associated with this technique is outlined in the literature. ${ }^{21,22}$ Small uncertainties were measured within the jet core near $(z=0$ $\mathrm{mm}$ ) since high signal-to-noise was observed in these areas. Since the fluorescence signal is proportional to the local concentration of NO, the signal-to-noise levels decreased at $z$ locations away from the jet core. Lower signal-tonoise decreases the accuracy of the technique as the ability to track the convection of the tagged lines becomes more difficult.

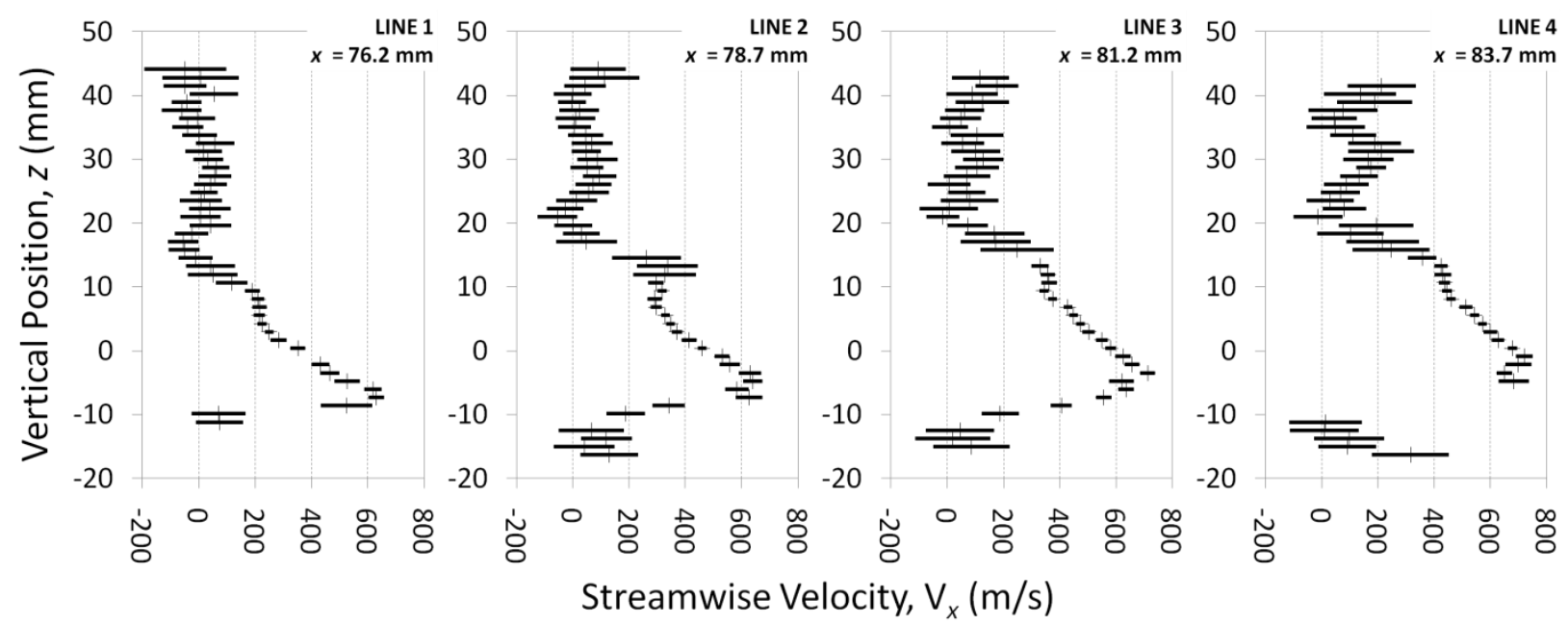

Figure 16: Vertical distribution $(z)$ of streamwise velocity $\left(V_{\mathrm{x}}\right)$. Jet $\mathrm{B} 2\left(\mathrm{P}_{\mathrm{c}}=128 \pm 2 \mathrm{psi}, \mathrm{P}_{0}=1301 \mathrm{psi}\right)$; AoA $=$ $-20^{\circ}$; Laser sheet at $y=-39 \mathrm{~mm}$. Width of bars indicates uncertainty in the mean values.

\section{Future Work}

The next major step of this work is to compare the experimental results to numerical predictions. A direct comparison between experimental and predicted velocity measurements would serve the basis of a validation for the computational code. In addition to the velocity measurements, the flow visualization images can be used to compare predictions of RCS jet trajectory, jet flow structure, and the three-dimensional separation structure in the wake. Upon validation, the numerical solutions can be used to further understand the main fluid dynamic problems such as flow separation, jet-crossflow interaction, and jet-jet interaction.

In terms of future flow visualization work, there are several interesting areas to investigate regarding the wakeflow and the interaction of the wake with the RCS jets. In the flow visualization portion of this study, NO was either seeded into the RCS jets or into the wake. As a result there was an absence of signal in both the outer-flow or within the shear layer. Additional seeding of NO from the heatshield forebody would allow PLIF measurements from these regions since a portion of the seeded NO gas would avoid entrainment into the capsule wake. It would also be interesting to study the Reynolds number effect on the capsule wake structure. In particular, the accuracy of the Reynolds number correlation on the separation angle put forth by Park et al. ${ }^{25}$ should be further investigated. Repetition of these measurements in a lower Mach number facility or changing the overall scale of the model would facilitate such a study on the Reynolds number dependence of hypersonic flow separation on practical entry vehicles.

Future flow visualization work focused on investigating the effects of the RCS jets on the three-dimensional wake structure should be undertaken. A comparison of wake-seeded tests with and without seeded RCS jets would assist in differentiating between fluorescence originating from the wake and fluorescence from the jets that penetrate into the outer flow. It would also be interesting to re-orient the laser sheet to study the development of streamwise vortical structures that form naturally in the wake and vortical structures that form from the presence of jets. Although the jet structures in the cross plane $(y-z)$ have been compiled from the current experimental data using the 
ViDI technique, re-orienting the laser sheet into the cross plane might reveal additional information. The advantage of obtaining single-shot images in the cross plane is that the spatial information would be correlated in time as opposed to the uncorrelated spatial information obtained from the compilation of single shot images performed in ViDI. Also absent from this study is the visualization of the RCS jet structure when the model is at a high angle of attack (i.e. $-25^{\circ}$ ). At high AoA the flow remains attached on the windward side of the aftbody surface and fluid ejected from the RCS jets interacts with an attached supersonic crossflow. In this case, the influence of a bow shock upstream of the RCS jet on the PLIF signal could be investigated. If the laser sheet was positioned through the center of the RCS jet, an angle-of-attack sweep would show instantaneous changes in the jet-in-crossflow structure as a transition from subsonic to supersonic crossflow would occur. Based on the results of this work, the sensitivity of the perceived jet spreading rate based on NO concentration levels is still unknown. A future study that systematically investigates the effects of $\mathrm{NO}$ concentration on the perceived jet structure produced after post processing would be useful.

There are several improvements and ideas for future work that could be undertaken with regards to the molecular tagging velocimetry measurements. Since the PLIF signal in the RCS jets overwhelmed the signal in the surrounding wake, a low gain setting was used on the camera system to avoid image saturation. If an order of magnitude reduction in NO concentration in the RCS jet were used in a future study, a more uniform signal level would be achieved. This would allow for higher camera gain settings, which would reduce the uncertainty of the velocity measurements in the separated wake flow away from the RCS jets. Reducing the temporal overlap of the $1^{\text {st }}$ exposure with the firing of the laser pulse, such that only a portion of the laser excitation were observed in the $1^{\text {st }}$ exposure, would also serve to reduce the amount of signal collected and allow for an increased gain setting. In the current experiment, the $1^{\text {st }}$ exposure captured the laser excitation over the duration of the pulse $(\sim 9.5 \mathrm{~ns})$ and subsequent fluorescence for a period of time after the pulse had ceased ( $\sim 5.5 \mathrm{~ns})$. Additionally, since the duration of the extent of the $1^{\text {st }}$ exposure beyond the end of the laser pulse was comparable to the duration of the pulse itself, use of the simple approximation for the temporal delay between the $1^{\text {st }}$ and $2^{\text {nd }}$ used in Ref. 22 may result in a small error. Although the magnitude of this error is expected to be negligible for the results presented in this study, future work aimed at quantifying the error is warranted. NO seeding from the vehicle forebody, as mentioned above, would allow velocity measurements to be obtained outside of the wake. Re-orientation of the MTV lines or the use of a cross-pattern of lines would allow a second component of velocity to be measured. Finally, orientation of the laser sheet into the cross plane would allow quantitative measurements of streamwise vorticity, which is expected to occur along the RCS jets trajectory and within the wake.

\section{Conclusion}

The wake flow of the MSL with multiple RCS jets was studied using the NO PLIF technique in NASA Langley Research Center's 31-Inch Mach 10 Air Tunnel facility. Image post-processing using the Virtual Diagnostics Interface (ViDI) method brought out the three dimensionality of the flow. Through an NO wake-seeding technique the three-dimensional separated region behind the capsule was visualized. The influence of model AoA on the separation region was also investigated in relation to the aerodynamic moments imposed on the vehicle. A critical separation angle was measured and compared to Reynolds-number-dependent empirical correlation developed for hypersonic flow separation. Comparison of wind-on and wind-off results illustrated the effects of jet-crossflow and jet-jet interactions, which occur in the wake of the MSL vehicle. Visualization of both single and multiple activated RCS jets indicate low levels of jet-jet interactions, which could degrade vehicle control authority. NO PLIF MTV was used to measure the streamwise component of velocity in the wake, indicating peak RCS jet velocities of up to $800 \mathrm{~m} / \mathrm{s}$. The MTV measurements provided a benchmark data set for numerical model validation.

\section{Acknowledgements}

We wish to acknowledge the contribution to this project from the NASA Langley Research Center 31-Inch Mach 10 Air Tunnel technicians and engineers, including Kevin Hollingsworth, Sheila Wright, Paul Tucker, Tony Robbins, Henry Fitzgerald and Johnny Ellis. Also, we wish to acknowledge those who designed the MSL model hardware and who organized and ran the wind tunnel test: Mark Schoenenberger, Matt Rhode, Vince Le Boffe and co-workers. In addition, we would like to acknowledge Andrew McCrea, who helped with the use of the ViDI software. This work was supported by the NASA Engineering and Safety Center (NESC) through Dave Schuster, Aerosciences Lead and the Aerodynamics-Aerothermodynamics-Plasma Discipline of the NASA Fundamental Aeronautics Program's Hypersonics Project with Deepak Bose as Technical Lead. Dr. Johansen was supported by the Natural Sciences and Engineering Research Council of Canada. 


\section{References}

${ }^{1}$ Dyakonov, A. A., Schoenenberger, M., Scallion, W. I., Van Norman, J. W., Novak, L. A., and Tang, C. Y. "Aerodynamic Interference Due to MSL Reaction Control System," AIAA Paper 2009-3915, 41 $1^{\text {st }}$ AIAA Thermophysics Conference, San Antonio, TX, June 22-25, 2009.

${ }^{2}$ Edquist, K. T., Dyakonov, A. A., Wright, M. J., and Tang, C. Y., "Aerothermodynamic Design of the Mars Science Laboratory Backshell and Parachute Cone," AIAA Paper 2009-4078 41 $1^{\text {st }}$ AIAA Thermophysics Conference, San Antonio, TX, June 22-25, 2009.

${ }^{3}$ Schoenenberger, M., Dyakonov, A. A., Buning, P. G., Scallion, W. I., and Van Norman, J., "Aerodynamic Challenges for the Mars Science Laboratory Entry, Descent and Landing," AIAA Paper 2009-3914, $41^{s t}$ AIAA Thermophysics Conference, San Antonio, TX, June 22-25, 2009.

${ }^{4}$ Dyakonov, A. A., Edquist, K. T., and Schoenenberger, M., "Influence of the Angle of Attack on the Aerothermodynamics of the Mars Science Laboratory," AIAA Paper 2009-3889, $36^{\text {th }}$ AIAA Fluid Dynamics Conference and Exhibit, San Francisco, CA, June 5-8, 2006.

5 Abilleira, F., "2011 Mars Science Laboratory Mission Design Overview," Earth and Space 2010 Conference, Honolulu, HI, March 14-17, 2010.

${ }^{6}$ Edquist, K. T., Dyakonov, A. A., Wright, M. J., Yang, C. Y., “Aerothermodynamic Environments Definition for the Mars Science Laboratory Entry Capsule," AIAA Paper 2007-1206, 45 ${ }^{\text {th }}$ AIAA Aerospace Sciences Meeting and Exhibit, Reno, NV, January 8-11, 2007

${ }^{7}$ Edquist, K. T., Liechty, D. S., Hollis, B. R., Alter, S. J., and Loomis, M. P., "Aeroheating Environments for a Mars Smart Lander," AIAA Paper 2002-4505, AIAA Atmospheric Flight Mechanics Conference and Exhibit, Monterey, CA, August 5-8, 2002.

${ }^{8}$ Schneider, S. P., "Laminar-Turbulent Transition on Reentry Capsules and Planetary Probes," Journal of Spacecraft and Rockets, Vol. 43, No. 6, 2006, pp. 1153-1173.

${ }^{9}$ Griffith, B. J., "Comparison of Aerodynamic Data from the Gemini Flights and AEDC-VKF Wind Tunnels," Journal of Spacecraft and Rockets, Vol. 4, No. 7, 1967, pp. 919-924.

${ }^{10}$ Sommer, S. C., Short, B. J., and Compton, D. L., "Free-Flight Measurements of Static and Dynamic Stability of Models of the Project Mercury Re-Entry Capsule at Mach Numbers 3 and 9.5" NASA TM-X-373, 1960.

${ }^{11}$ Ben-Yakar, A., Mungal, G. M., and Hanson, R. K., "Time Evolution and Mixing Characteristics of Hydrogen and Ethylene Transverse Jets in Supersonic Crossflows," Physics of Fluids, Vol. 18, No. 2, 2006, Paper 026101.

${ }^{12}$ Gruber, M. R., Nejad, A. S., Chen, T. H., and Dutton, J. C., "Mixing and Penetration Studies of Sonic Jets in a Mach 2 Freestream, ” Journal of Propulsion and Power, Vol. 11, No. 2, 1995, pp. 315-323.

${ }^{13}$ Wright, M.J., Tang, C.Y., Edquist, K.T., Hollis, B.R., Krasa, P., and Campbell, C.A., "A Review of Aerothermal Modeling for Mars Entry Missions," AIAA Paper 2010-0443, 48 $8^{\text {th }}$ AIAA Aerospace Sciences Meeting Including the New Horizons Forum and Aerospace Exposition, Orlando, FL, January 4-7, 2010.

${ }^{14}$ Inman, J. A., Danehy, P. M., Alderfer, D. W., Buck, G. M., and McCrea, A., "Planar Fluorescence Imaging and Three Dimensional Reconstructions of Capsule Reaction-Control-Systems Jets," AIAA Journal, Vol. 47, No. 4, 2009, pp. 803-812.

${ }^{15}$ Danehy, P. M., Inman, J. A., Brauckmann, G., Alderfer, D. W., Johnse, S. B., and Patry, D., "Visualization of a Capsule Entry Vehicle Reaction-Control System Thruster," Journal of Spacecraft and Rockets, Vol. 46, No. 1, 2009, pp. 93-102.

${ }_{16}$ Micol, J. R., "Langley Aerothermodynamic Facilities Complex: Enhancements and Testing Capabilities," AIAA Paper 98-0147, 36th AIAA Aerospace Sciences Meeting and Exhibit, Reno, NV, January 12-15, 1998.

17 Danehy, P. M., Inman, J. A., Alderfer, D. W., Buck, G. M., and Bathel, B. F., "Visualization of flowfield modification by RCS jets on a capsule entry vehicle", AIAA Paper 2008-1231, 46th AIAA Aerospace Sciences Meeting and Exhibit, Reno, NV, Jan. 7-10, 2008.

${ }_{18}$ Danehy, P. M., Alderfer, D. W., Inman, J. A., Berger, K. T., Buck, G. M., and Schwartz, R. J., "Fluorescence Imaging and Streamline Visualization of Hypersonic Flow Over Rapid Prototype Wind-Tunnel Models" Proceedings of the Institution of Mechanical Engineers - Part G: Journal of Aerospace Engineering, Vol. 222 (G5), 2008, pp. 637-651.

${ }^{19}$ Sorzano, C. O., Thevenaz, P., and Unser, M., "Elastic Registration of Biological Images Using Vector-Spline Regularization," IEEE Transactions on Biomedical Engineering, Vol. 52, No. 4, 2005, pp. 652-663.

${ }^{20}$ Rasband, W. S., ImageJ, U. S. National Institutes of Health, Bethesda, MD, USA, http://rsb.info.nih.gov/ij, 1997-2009. 
${ }^{21}$ Bathel, B. F., Danehy, P. M., Inman, J. A., Jones, S. B., Ivey, C. B. and Goyne, C. P., “ Multiple Velocity Profile Measurements in Hypersonic Flows Using Sequentially-Imaged Fluorescence Tagging," AIAA Paper 20101404, 48 ${ }^{\text {th }}$ AIAA Aerospace Sciences Meeting, Orlando, FL, January 4-7, 2010.

${ }^{22}$ Bathel, B. F., Danehy, P. M., Inman, J. A., Jones, S. B., Ivey, C. B., and Goyne, C. P. "Velocity Profile Measurements in Hypersonic Flows Using Sequentially-Imaged Fluorescence-Based Molecular Tagging Velocimetry," AIAA Journal (to be published).

${ }^{23}$ Bathel, B. F., Danehy, P. M., Inman, J. A., Watkins, A. N., Jones, S. B., Lipford, W. E., Goodman, K. Z., Ivey, C. B., and Goyne, C. P., "Hypersonic Laminar Boundary Layer Velocimetry with Discrete Roughness on a Flat Plate," AIAA Paper 2010-4998, 40th Fluid Dynamics Conference and Exhibit, Chicago, IL, June 28 - July 1, 2010.

${ }^{24}$ Lamb, J. P., and Oberkampf, W. L., "Review and Development of Base Pressure and Base Heating Correlations in Supersonic Flow," Journal of Spacecraft and Rockets, Vol. 32, No. 1, 1995, pp. 8-23.

${ }^{25}$ Park, G., Gai, S. L., and Neely, A. J., "Laminar Near Wake of a Circular Cylinder at Hypersonic Speeds," AIAA Journal, Vol. 48, No. 1, 2010, pp. 236-248.

${ }^{26}$ Park, G., private communication, May 2011. 